\title{
Random Assignment Versus Fixed Assignment in Multilevel Importance Splitting for Estimating Stochastic Reach Probabilities
}

\author{
Hao Ma ${ }^{1,2}$ (D) Henk A. P. Blom ${ }^{1}$ (D)
}

Received: 25 October 2020 / Revised: 2 June 2021 / Accepted: 17 August 2021/

Published online: 28 January 2022

(C) The Author(s) 2022

\begin{abstract}
This paper focuses on estimating reach probability of a closed unsafe set by a stochastic process. A well-developed approach is to make use of multi-level MC simulation, which consists of encapsulating the unsafe set by a sequence of increasing closed sets and conducting a sequence of MC simulations to estimate the reach probability of each inner set from the previous set. An essential step is to copy (split) particles that have reached the next level (inner set) prior to conducting a MC simulation to the next level. The aim of this paper is to prove that the variance of the multi-level MC estimated reach probability under fixed assignment splitting is smaller or equal than under random assignment splitting methods. The approaches are illustrated for a geometric Brownian motion example.
\end{abstract}

Keywords Multilevel importance splitting · Interacting particles · Reach probability · Monte Carlo method · Multi-dimensional diffusion process

\section{Introduction}

Evaluating the reach probability of an unsafe set is well-studied in the domains of control and safety verification of complex safety critical system designs. In the control domain the focus is on synthesizing a control policy such that a safety critical system stays away from the unsafe set with a high probability (Alur et al. 2000; Prandini and $\mathrm{Hu}$ 2007). From this control synthesis perspective it makes good sense to adopt model abstractions in combination with an over-approximation of the unsafe set (Julius and Pappas 2009; Abate et al. 2011; Di Benedetto et al. 2015). In safety verification of complex safety critical system design reach probability of the unsafe set is commonly evaluated using statistical simulation techniques, e.g. air traffic (Blom et al. 2006, 2007),

\section{Hao Ma}

Henk A. P. Blom

h.a.p.blom@tudelft.nl

1 Delft University of Technology, 2629 HS Delft, Zuid Holland, The Netherlands

2 Northwestern Polytechnical University, Xi'an 710072, China 
actuarial risks (Asmussen and Albrecher 2010), random graphs (Bollobás 2001), communication network reliability (Robert 2003).

To evaluate very small reach probabilities, common practice is to make use of methods to reduce variance for a given computational effort. Literature on variance reduction distinguishes two main approaches: Importance Sampling (IS) and Importance Splitting (ISp). IS draws samples from a reference stochastic system model in combination with an analytical compensation for sampling from the reference model instead of the intended model. Bucklew (2004) gives an overview of IS and analytical compensation mechanisms. For complex models analytical compensation mechanisms typically fall short and multi-level ISp is the preferred approach (e.g. Botev and Kroese 2008; L'Ecuyer et al. 2009; Rubinstein 2010; Morio and Balesdent 2016).

In multi-level splitting the safe set, or target set, i.e. the set for which the reach probability has to be estimated, is enclosed by a series of strictly increasingly subsets. This multi-level setting allows to express the small reach probability of the inner level set as a product of larger reach probabilities for the sequence of enclosing subsets (see e.g. Glasserman et al. 1998, 1999). Cérou et al. (2005, 2006) embedded this multi-level splitting in the Feynman-Kac factorization equation for strong Markov processes (Del Moral 2004). This Feynman-Kac setting subsequently supported the evaluation of the reach probability through sequential Monte Carlo simulation in the form of an Interacting Particle System (IPS), including characterization of asymptotic behaviour (Cérou et al. 2006).

Particle splitting (copying) of $N_{S}$ successful particles to $N_{P} \geq N_{S}$ particles can be done in multiple ways (e.g. Garvels and Kroese 1998; Cérou et al. 2006; L'Ecuyer et al. 2007; L'Ecuyer et al. 2009). The classical approach is Multinomial Resampling, i.e. drawing the $N_{P}$ particles at random, with replacement, from the $N_{S}$ successful particles. Cérou et al. (2006) propose the alternative of adding to the set of $N_{S}$ successful particles, $N_{P}-N_{S}$ random drawings (with replacement) from the $N_{S}$ successful particles; this we refer to as Multinomial Splitting. A third approach is fixed assignment splitting, i.e. copying each of the $N_{S}$ successful particles as much as possible the same number of times. Following (L'Equyer et al. 2009) fixed assignment splitting is accomplished in two steps. During the first step each successful particle is copied $\left\lfloor N_{P} / N_{S}\right\rfloor$ times. During the second step, the residual $N_{P}-N_{S}\left\lfloor N_{P} / N_{S}\right\rfloor$ particles are randomly chosen (without replacement) from the set of successful particles, and these are added to the set of copies from the first step. A fourth approach is residual multinomial splitting, i.e. after the first step of fixed assignment splitting, the residual $N_{P}-N_{S}\left\lfloor N_{P} / N_{S}\right\rfloor$ particles are randomly chosen (with replacement) from the $N_{S}$ successful particles.

Under restrictive assumptions Garvels (2000) has proven that fixed assignment splitting works better or equal to multinomial resampling. The key assumption is that the sets of particles at different levels are independent from each other. In IPS for filtering studies, e.g. (Del Moral et al. 2001; Gerber et al. 2019), multi-level Feynman-Kac analysis has been used to make variance comparisons between different particle resampling methods. Through mapping the filtering IPS results of Del Moral et al. (2001) to the reach probability IPS, Cérou et al. (2006) argue that multinominal resampling adds extra randomness to multinomial splitting, as a result of which the multinomial splitting has a variance advantage over multinomial resampling. Through mapping the filtering IPS results of Gerber et al. (2019) to the reach probability IPS, it is clear that residual multinomial splitting has a variance advantage over multinomial resampling. Gerber et al. (2019) also conclude that existing multi-level Feynman-Kac analysis falls short in handling random drawings without replacement, as is done in the second step of fixed assignment splitting. 
The main objective of this paper is to prove that using fixed assignment splitting in reach probability IPS dominates in variance reduction over the random assignment methods: Multinomial Resampling, Multinomial Splitting and Residual Multinomial Splitting. These proofs do not make use of the independence assumption of Garvels (2000). The stochastic process considered is a multi-dimensional diffusion process that is pathwise continuous. The effect of different splitting methods is also illustrated in reach probability estimation for a geometric Brownian motion example.

This paper is organized as follows. Section 2 reviews the background of IPS based reach probability estimation for a multi-dimensional diffusion process. Section 3 characterizes the conditional variances of IPS based reach probability estimation under multinominal resampling, multinominal splitting, residual multinominal splitting and fixed assignment splitting. Section 5 proofs that fixed assignment splitting has a variance advantage over these other three ways of splitting. Section 6. presents a case study based on a geometric Brownian motion for evaluating and comparing multinominal resampling, multinomial splitting and fixed assignment splitting. Section 6 draws conclusions.

\section{IPS based Reach Probability Estimation}

\subsection{Reach Probability of Multi-dimensional Diffusion}

For the rest of the paper, we define all stochastic processes on a complete probability space $(\Omega, F, \mathrm{P})$. The problem is to estimate the probability $\gamma$ that a $\mathbb{R}^{n}$-valued pathwise continuous diffusion process $\left\{x_{t}\right\}$ reaches a closed subset $D \subset \mathbb{R}^{n}$ within finite period $[0, T]$, i.e.

$$
\gamma=P(\tau<T)
$$

with $\tau$ the first hitting time of $D$ by $\left\{x_{t}\right\}$ :

$$
\tau=\inf \left\{t>0, x_{t} \in D\right\}
$$

Remark Cérou et al. (2006) and L'Equyer et al. (2009) also address the more general situation that $T$ is a $\mathrm{P}$-a.s. finite stopping time.

\subsection{Multi-level Factorization}

If the reach probability $\gamma$ in (1) is too small, then a straightforward MC estimator requires a considerable amount of samples. To overcome this, we introduce a nested sequence of closed subsets $D_{k}$ of $\mathbb{R}^{n}$ to factorize the reach probability $\gamma$, such that $D=D_{m} \subset D_{m-1} \subset \cdots \subset D_{1} \subset \mathbb{R}^{n}$ and $P\left\{x_{0} \in D_{1}\right\}=0$. Let $\tau_{k}$ be the first moment in time that $\left\{x_{t}\right\}$ reaches $D_{k}$, i.e.

$$
\tau_{k}=\inf \left\{t>0 ; x_{t} \in D_{k} \vee t \geq T\right\}
$$

Then, we define $\{0,1\}$-valued random variables $\left\{\chi_{k}, k=1, . ., m\right\}$ as follows:

$$
\begin{aligned}
\chi_{k} & =1, \text { if } \tau_{k}<T \text { or } k=0 \\
& =0, \text { else }
\end{aligned}
$$

By using this $\chi_{k}$ definition, the factorization becomes: 


$$
\gamma=\prod_{k=1}^{m} \gamma_{k}
$$

with $\gamma_{k} \triangleq \mathrm{P}\left(\chi_{k}=1 \mid \chi_{k-1}=1\right)=\mathrm{P}\left(\tau_{k}<T \mid \tau_{k-1}<T\right)$.

\subsection{Recursive Estimation of the Multi-level Factors}

By using the strong Markov property of $\left\{x_{t}\right\}$, we can develop a recursive estimation of $\gamma$ using the factorization in (5). First, we define $\xi_{k} \triangleq\left(\tau_{k}, x_{\tau_{k}}\right), Q_{k} \triangleq(0, T) \times D_{k}$, for $k=1, \ldots, m$, and the following conditional probability measure $\pi_{k}(B)$ for an arbitrary Borel set $B$ of $\mathbb{R}^{n+1}$ :

$$
\pi_{k}(B) \triangleq P\left(\xi_{k} \in B \mid \xi_{k} \in Q_{k}\right)
$$

Cérou et al. (2006) show that $\pi_{k}$ is a solution of the following recursion of transformations:

$$
\pi_{k-1}(\cdot) \stackrel{\text { I. mutation }}{\longrightarrow} p_{k}(\cdot) \stackrel{\text { III. selection }}{\longrightarrow} \pi_{k}(\cdot)
$$

where $p_{k}(B)$ is the conditional probability measure of $\xi_{k} \in B$ given $\xi_{k-1} \in Q_{k-1}$ i.e.

$$
p_{k}(B) \triangleq P\left(\xi_{k} \in B \mid \xi_{k-1} \in Q_{k-1}\right)
$$

Because $\left\{x_{t}\right\}$ is a strong Markov process, $\left\{\xi_{k}\right\}$ is a Markov sequence. Therefore the mutation transformation (I) satisfies a Chapman-Kolmogorov equation prediction for $\xi_{k}$ :

$$
p_{k}(B)=\int_{E^{\prime}} p_{\xi_{k} \mid \xi_{k-1}}(B \mid \xi) \pi_{k-1}(d \xi) \text { for all } B \in \beta\left(\mathbb{R}^{n+1}\right)
$$

For the conditioning transformation (II) this means:

$$
\gamma_{k}=\mathrm{P}\left(\tau_{k}<T \mid \tau_{k-1}<T\right)=\int_{E^{\prime}} 1_{\left\{\xi \in Q_{k}\right\}} p_{k}(d \xi) .
$$

Hence, selection transformation (III) satisfies:

$$
\pi_{k}(B)=\frac{\int_{B} 1_{\left\{\xi \in Q_{k}\right\}} p_{k}(d \xi)}{\int_{E^{\prime}} 1_{\left\{\xi^{\prime} \in Q_{k}\right\}} p_{k}\left(d \xi^{\prime}\right)}=\left[\int_{B} 1_{\left\{\xi \in Q_{k}\right\}} p_{k}(d \xi)\right] / \gamma_{k}
$$

With this, the $\gamma_{k}$ terms in (5) are characterized as solutions of a recursive sequence of mutation equation (6), conditioning equation (7), and selection equation (8).

\subsection{IPS Algorithmic Steps}

Following Cérou et al. (2006), eqs. (5)-(8) yield the IPS algorithmic steps for the numerical estimation of $\gamma$ : 


$$
\begin{gathered}
\bar{\pi}_{k-1}(\cdot) \stackrel{\text { I. mutation }}{\longrightarrow} \bar{p}_{k}(\cdot) \stackrel{\text { III. selection }}{\longrightarrow} \tilde{\pi}_{k}(\cdot) \stackrel{\text { IV. splitting }}{\longrightarrow} \bar{\pi}_{k}(\cdot) \\
\\
\bar{\gamma}_{k} \text { II. conditioning }
\end{gathered}
$$

A set of $N_{P}$ particles is used to form empirical density approximations $\bar{\gamma}_{k}, \bar{p}_{k}$ and $\gamma_{k}, \bar{\pi}_{k}$ of $p_{k}$ and $\pi_{k}$ respectively. By increasing the number $N_{P}$ of particles in a set, the errors in these approximations will decrease. When simulating particles from $Q_{k-1}$ to $Q_{k}$, a fraction $\bar{\gamma}_{k}$ of the simulated particle trajectories only will reach $Q_{k}$ within the time period $[0, T]$ considered; these particles form $\tilde{\pi}_{k}$. Prior to starting the next IPS cycle with $N_{P}$ particles, $\left(N_{P}-N_{S_{k}}\right.$ ) copies (also called splittings) from the $N_{S_{k}}$ successful particles in $\tilde{\pi}_{k}$ are added to $\bar{\pi}_{k}$. In the next sections we consider four ways of splitting: multinominal resampling, multinominal splitting, residual multinominal splitting and fixed assignment splitting.

Under Multinomial Splitting, Cérou et al. (2006) prove that $\bar{\gamma}$ forms an unbiased $\gamma$ estimate, i.e.

$$
\mathbb{E}\{\bar{\gamma}\}=\mathbb{E}\left\{\prod_{k=1}^{m} \bar{\gamma}_{k}\right\}=\prod_{k=1}^{m} \mathbb{E}\left\{\bar{\gamma}_{k}\right\}=\prod_{k=1}^{m} \gamma_{k}=\gamma
$$

Moreover, Cérou et al. (2006) derive second and higher order asymptotic bounds for the error $(\bar{\gamma}-\gamma)$ based on multi-level Feynman Kac analysis, e.g. Del Moral (2004; Theorem 12.2.2).

\section{CONDITIONAL VARIANCE CHARACTERIZATIONS}

In this section, conditional characterizations of the variance of $\bar{\gamma}_{k}$ are developed for IPS using multinomial resampling (MR), multinomial splitting (MS), residual multinomial splitting (RMS) and fixed assignment splitting (FAS), respectively.

\subsection{IPS using Multinomial Resampling}

In IPS using multinomial resampling, $N_{P}$ offspring are cloned randomly from $\tilde{\pi}_{k}$. The resulting algorithm of IPS with multinomial resampling, starting from $\xi_{k-1}^{i}$, is described in Algorithm 1 below. 


\section{Algorithm 1. IPS using multinomial resampling}

Input: Initial measure $\pi_{0}$, end time $T$, decreasing sequence of closed subsets $D_{k}=\left\{x_{t} \in \mathbb{R}^{n}\right\}, D_{k-1} \supset D_{k}, k=1, . ., m$. Also $D_{0}=\mathbb{R}^{n}, Q_{k}=(0, T) \times D_{k}$ and number of particles $N_{P}$.

Output: Estimated reach probability $\bar{\gamma}$ collects

0 . Initiation: Generate $N_{P}$ particles $\xi_{0}^{i} \sim \pi_{0}, i=1, \ldots, N_{P}$, i.e., $\bar{\pi}_{0}(\cdot)=\sum_{i=1}^{N_{p}} \frac{1}{N_{p}} \delta_{\left\{\xi_{0}^{i}\right\}}(\cdot)$, with Dirac $\delta$. Set $k=1$.

I. Mutation: $\bar{p}_{k}()=.\sum_{i=1}^{N_{p}} \frac{1}{N_{p}} \delta_{\left\{\bar{\xi}_{k}^{i}\right\}}(\cdot)$, where $\bar{\xi}_{k}^{i}$ is obtained through simulating the strong Markov process starting from $\xi_{k-1}^{i}$.

II. Conditioning: $N_{S_{k}}=\sum_{i=1}^{N_{p}} 1\left(\bar{\xi}_{k}^{i} \in Q_{k}\right)$ and $\bar{\gamma}_{k}=\frac{N_{S_{k}}}{N_{p}}$.

If $N_{S_{k}}=0$, then $\bar{\gamma}_{k^{\prime}}=0, k^{\prime} \in\{k, . ., m\}$ and go to Step V.

III. Selection: $\tilde{\pi}_{k}()=.\frac{1}{N_{S_{k}}} \sum_{i=1}^{N_{S_{k}}} \delta_{\left\{\tilde{\xi}^{i}\right\}}(\cdot)$, with $\left\{\tilde{\xi}_{k}^{j}\right\}_{j=1}^{N_{S_{k}}}$ the collection of $\bar{\xi}_{k}^{i} \in Q_{k}, i=1, . ., N_{P}$.

IV. Splitting: $\bar{\pi}_{k}()=.\sum_{i=1}^{N_{p}} \frac{1}{N_{p}} \delta_{\left\{\xi_{k}^{i}\right\}}($.$) , with \xi_{k}^{i} \sim \tilde{\pi}_{k}($.$) .$

V. If $k<m$, then $k:=k+1$ and go to Step I, else $\bar{\gamma}=\prod_{k=1}^{m} \bar{\gamma}_{k}$

In order to gain a better understanding of the probabilistic characteristics of the particles that reached a level, we now characterize the conditional distribution of particles that reach level $k+1$, given that at level $k$ the $i$-th successful particle $\tilde{\xi}_{k}^{i}$ is copied $K_{k}^{i}$ times, $i=1, . ., N_{S_{k}}$.

Proposition 1: If $N_{S_{k}}>0$ and $K_{k}^{i}$, with $i=1,2, \ldots, N_{S_{k}}$, denote the number of particles that copies $\tilde{\xi}_{k}^{i}$ at level $k$. Then the number $Y_{k+1}^{k, i}$, of the $K_{k}^{l}$ particle copies of $\tilde{\xi}_{k}^{i}$ that reach level $k+1$, has a conditional Binomial distribution of size $K_{k}^{i}$ and success probability $\gamma_{k+1}\left(\tilde{\xi}_{k}^{i}\right)$, i.e.

$$
p_{Y_{k+1}^{k, i} \mid K_{k}^{i}, \tilde{\xi}_{k}^{i}}\left(n ; K_{k}^{i}, \tilde{\xi}_{k}^{i}\right)=\operatorname{Bin}\left(n ; K_{k}^{i}, \gamma_{k+1}\left(\tilde{\xi}_{k}^{i}\right)\right)
$$

with

$$
\gamma_{k+1}\left(\tilde{\xi}_{k}^{i}\right) \triangleq \mathrm{P}\left(\tau_{k+1}<T \mid \xi_{k}=\tilde{\xi}_{k}^{i}\right)
$$

Proof: See 8 .. 
Theorem 1: If $N_{S_{k}} \geq 1$ and $K_{k}^{i}, i=1, . . N_{S_{k}}$, denotes the number of copies made of the $i$-th successful particle $\tilde{\xi}_{k}^{i}$ during the splitting step at level $k$ of the IPS algorithm, then.

$$
\begin{gathered}
\mathbb{E}\left\{\bar{\gamma}_{k+1} \mid \tilde{\xi}_{k}^{j}, \text { all } j\right\}=\frac{1}{N_{p}} \sum_{i=1}^{N_{S_{k}}}\left[\mathbb{E}\left\{K_{k}^{i} \mid \tilde{\xi}_{k}^{j}, \text { all } j\right\} \gamma_{k+1}\left(\tilde{\xi}_{k}^{i}\right)\right] \\
\operatorname{Var}\left\{\bar{\gamma}_{k+1} \mid \tilde{\xi}_{k}^{j}, \text { all } j\right\} \\
=\frac{1}{N_{p}^{2}} \sum_{i=1}^{N_{S_{k}}}\left[\mathbb{E}\left\{K_{k}^{i} \mid \tilde{\xi}_{k}^{j}, \text { all } j\right\} \gamma_{k+1}\left(\tilde{\xi}_{k}^{i}\right)\left(1-\gamma_{k+1}\left(\tilde{\xi}_{k}^{i}\right)\right)\right] \\
+\frac{1}{N_{p}^{2}} \sum_{i=1}^{N_{S_{k}}}\left[\operatorname{Var}\left\{K_{k}^{i} \mid \tilde{\xi}_{k}^{j}, \text { all } j\right\} \gamma_{k+1}\left(\tilde{\xi}_{k}^{i}\right)^{2}\right] \\
+\frac{1}{N_{p}^{2}} \sum_{i=1}^{N_{S_{k}}} \sum_{\substack{i_{S_{k}} \\
i^{\prime}=1}}^{i^{\prime} \neq i}\left[\operatorname{Cov}\left\{K_{k}^{i}, K_{k}^{i^{\prime}} \mid \tilde{\xi}_{k}^{j}, \text { all } j\right\} \gamma_{k+1}\left(\tilde{\xi}_{k}^{i}\right) \gamma_{k+1}\left(\tilde{\xi}_{k}^{i^{\prime}}\right)\right]
\end{gathered}
$$

Proof: See 8 ..

Proposition 2: If $N_{S_{k}} \geq 1$, and we use multinomial resampling at IPS level $k$ then.

$$
\begin{gathered}
\mathbb{E}\left\{\bar{\gamma}_{k+1} \mid \tilde{\xi}_{k}^{1}, \ldots, \tilde{\xi}_{k}^{N_{S_{k}}}\right\}=\frac{1}{N_{S_{k}}} \sum_{i=1}^{N_{S_{k}}} \gamma_{k+1}\left(\tilde{\xi}_{k}^{i}\right) \\
\operatorname{Var}\left\{\bar{\gamma}_{k+1} \mid \tilde{\xi}_{k}^{1}, \ldots, \tilde{\xi}_{k}^{N_{S_{k}}}\right\} \\
=\frac{1}{N_{p} N_{S_{k}}} \sum_{i=1}^{N_{S_{k}}}\left[\gamma_{k+1}\left(\tilde{\xi}_{k}^{i}\right)\left(1-\gamma_{k+1}\left(\tilde{\xi}_{k}^{i}\right)\right)\right] \\
+\frac{1}{N_{p} N_{S_{k}}}\left[\sum_{i=1}^{N_{S_{k}}}\left[\left(\gamma_{k+1}\left(\tilde{\xi}_{k}^{i}\right)\right)^{2}\right]-\frac{1}{N_{S_{k}}} \sum_{i=1}^{N_{S_{k}}} \sum_{i^{\prime}=1}^{N_{S_{k}}}\left[\left(\gamma_{k+1}\left(\tilde{\xi}_{k}^{i}\right) \gamma_{k+1}\left(\tilde{\xi}_{k}^{i^{\prime}}\right)\right)\right]\right.
\end{gathered}
$$

Proof: See 8..

\subsection{IPS using multinomial splitting}

IPS using multinomial splitting follows the steps of Algorithm 1, except for splitting step IV. Now each particle in $\tilde{\pi}_{k}(\cdot)$ is first copied once, and then $\left(N_{p}-N_{S_{k}}\right)$ offspring are cloned randomly from $\tilde{\pi}_{k}(\cdot)($ Cérou et al. 2006, Sect. 3.2, p. 189). This multinomial splitting in IPS step IV is specified in Algorithm 2 


\section{Algorithm 2. Multinomial Splitting in IPS step IV}

IV. Splitting: $\xi_{k}^{i}=\tilde{\xi}_{k}^{i}$ for $i=1, \ldots, N_{S_{k}}$; then $\xi_{k}^{i} \sim \tilde{\pi}_{k}($.$) for$

$$
i=N_{S_{k}}+1, \ldots, N_{p} \text {. }
$$

Each particle receives weight $1 / N_{p}$.

In IPS using multinomial splitting, all particles have the same weight at any given level. Each particle is simulated until it reaches the first subset $Q_{1}$. Then $\sum_{i=1}^{N_{p}} 1\left(\bar{\xi}_{1}^{i} \in Q_{1}\right)$ is the number of particles that have reached the first subset $Q_{1}$. The fraction $\bar{\gamma}_{1}=\sum_{i=1}^{N_{p}} \frac{1}{N_{p}} 1\left(\bar{\xi}_{1}^{i} \in Q_{1}\right)$ is an unbiased estimate of $\gamma_{1}=\mathrm{P}\left(\tau_{1}<T\right)$. To maintain a sufficiently large population of particles, in IPS step IV $\left(N_{P}-N_{S_{k}}\right)$ copies of these $\sum_{i=1}^{N_{p}} 1\left(\bar{\xi}_{1}^{l} \in Q_{1}\right)$ successful particles are added to the set of $N_{S_{k}}$ successful particles. During the next IPS cycle each new particle is simulated until it reaches the second subset $Q_{2}$. Again, the fraction $\bar{\gamma}_{2}=\sum_{i=1}^{N_{p}} \frac{1}{N_{p}} 1\left(\bar{\xi}_{2}^{i} \in Q_{2}\right)$ of $\sum_{i=1}^{N_{p}} 1\left(\bar{\xi}_{2}^{i} \in Q_{2}\right)$ particles that reach the second subset $Q_{2}$ is a natural estimate of $\gamma_{2}=\mathrm{P}\left(\tau_{2}<T \mid \tau_{1}<T\right)$. This cycle is repeated until particles reach the last subset $Q_{m}$. The fraction $\bar{\gamma}_{k}=\sum_{i=1}^{N_{p}} \frac{1}{N_{p}} 1\left(\bar{\xi}_{k}^{i} \in Q_{k}\right)$ of particles that have timely reached the $k$-th subset from the preceding subset is an unbiased estimate of $\gamma_{k}=\mathrm{P}\left(\tau_{k}<T \mid \tau_{k-1}<T\right)$. From Eq. (9) we know that the product of these $m$ fractions is an unbiased estimate of $\gamma=\mathrm{P}\left(\tau_{m}<T\right)$.

It is straightforward to verify that Proposition 1 and Theorem 1 also hold true using multinomial splitting in IPS step IV.

Proposition 3: If $N_{S_{k}} \geq 1$, and we use multinomial splitting at IPS level $k$ then.

$$
\begin{aligned}
& \mathbb{E}\left\{\bar{\gamma}_{k+1} \mid \tilde{\xi}_{k}^{1}, \ldots, \tilde{\xi}_{k}^{N_{S_{k}}}\right\}=\frac{1}{N_{S_{k}}} \sum_{i=1}^{N_{S_{k}}} \gamma_{k+1}\left(\tilde{\xi}_{k}^{i}\right) \\
& \operatorname{Var}\left\{\bar{\gamma}_{k+1} \mid \tilde{\xi}_{k}^{1}, \ldots, \tilde{\xi}_{k}^{N_{S_{k}}}\right\} \\
& =\frac{1}{N_{p} N_{S_{k}}} \sum_{i=1}^{N_{S_{k}}}\left[\gamma_{k+1}\left(\tilde{\xi}_{k}^{i}\right)\left(1-\gamma_{k+1}\left(\tilde{\xi}_{k}^{i}\right)\right)\right] \\
& +\frac{\left(N_{p}-N_{S_{k}}\right)}{N_{p}^{2} N_{S_{k}}}\left[\sum_{i=1}^{N_{S_{k}}}\left[\left(\gamma_{k+1}\left(\tilde{\xi}_{k}^{i}\right)\right)^{2}\right]-\frac{1}{N_{S_{k}}} \sum_{i=1}^{N_{S_{k}}} \sum_{i^{\prime}=1}^{N_{S_{k}}}\left[\gamma_{k+1}\left(\tilde{\xi}_{k}^{i}\right) \gamma_{k+1}\left(\tilde{\xi}_{k}^{i^{\prime}}\right)\right]\right]
\end{aligned}
$$

Proof: See $8 .$.

\subsection{IPS Using Residual Multinomial Splitting}

IPS using residual multinomial splitting follows the steps of Algorithm 1 with a new Step IV. Now each successful particle is first copied $\alpha_{k}=\left\lfloor N_{p} / N_{S_{k}}\right\rfloor$ times, and then 
residual $\left(N_{p} \bmod N_{S_{k}}\right)$ particles are randomly drawn from $\tilde{\pi}_{k}(\cdot)$. The residual multinomial splitting step IV is specified in Algorithm 3 below.

\section{Algorithm 3. Residual multinomial splitting in IPS step IV}

$$
\begin{aligned}
& \text { IV. Splitting: } \xi_{k}^{i}=\tilde{\xi}_{k}^{i} \text { for } i=1, \ldots, N_{S_{k}} \text {; } \\
& \xi_{k}^{i+N_{S_{k}}}=\widetilde{\xi}_{k}^{i} \text { for } i=1, \ldots, N_{S_{k}} ; \\
& \text {... } \\
& \xi_{k}^{i+\left[\frac{N_{p}}{N_{S_{k}}}-1\right\rfloor_{N_{S_{k}}}}=\tilde{\xi}_{k}^{i} \text { for } i=1, \ldots, N_{S_{k}} ; \\
& \xi_{k}{ }^{\left.i+\mid \frac{N_{p}}{N_{S_{k}}}\right\rfloor N_{S_{k}}} \sim \tilde{\pi}_{k}(.) \text { for } i=1, \ldots, N_{p}-\left\lfloor\frac{N_{p}}{N_{S_{k}}}\right\rfloor N_{S_{k}} .
\end{aligned}
$$

Each particle receives weight $1 / N_{p}$.

Straightforward verification shows that Proposition 1 and Theorem 1 also hold true when using residual multinomial splitting in IPS step IV.

Proposition 4: If $N_{S_{k}} \geq 1$, and we use residual multinomial splitting at IPS level $k$ then.

$$
\begin{gathered}
\mathbb{E}\left\{\bar{\gamma}_{k+1} \mid \tilde{\xi}_{k}^{1}, \ldots, \tilde{\xi}_{k}^{N_{S_{k}}}\right\}=\frac{1}{N_{S_{k}}} \sum_{i=1}^{N_{S_{k}}} \gamma_{k+1}\left(\tilde{\xi}_{k}^{i}\right) \\
\operatorname{Var}\left\{\bar{\gamma}_{k+1} \mid \tilde{\xi}_{k}^{1}, \ldots, \tilde{\xi}_{k}^{N_{S_{k}}}\right\} \\
=\frac{1}{N_{p} N_{S_{k}}} \sum_{i=1}^{N_{S_{k}}}\left[\gamma_{k+1}\left(\tilde{\xi}_{k}^{i}\right)\left(1-\gamma_{k+1}\left(\tilde{\xi}_{k}^{i}\right)\right)\right]+\frac{\left(N_{p} \bmod N_{S_{k}}\right)}{N_{p}^{2} N_{S_{k}}} \\
\cdot\left[\sum_{i=1}^{N_{S_{k}}}\left[\left(\gamma_{k+1}\left(\tilde{\xi}_{k}^{i}\right)\right)^{2}\right]-\frac{1}{N_{S_{k}}} \sum_{i=1}^{N_{S_{k}}} \sum_{i^{\prime}=1}^{N_{S_{k}}}\left[\left(\gamma_{k+1}\left(\tilde{\xi}_{k}^{i}\right) \gamma_{k+1}\left(\tilde{\xi}_{k}^{i^{\prime}}\right)\right)\right]\right]
\end{gathered}
$$

Proof: See 8.

\subsection{IPS Using Fixed Assignment Splitting}

When using fixed assignment splitting, each particle in $\tilde{\pi}_{k}(\cdot)$ is copied as much as possible the same number of times. This is applied by first copying each particle $\left\lfloor N_{p} / N_{S_{k}}\right\rfloor$ times, and then making $\left(N_{p} \bmod N_{S_{k}}\right)$ copies from distinct particles chosen at random (without replacement). So the chosen particles would be copied $\left\lfloor N_{p} / N_{S_{k}}\right\rfloor+1$ times (L'Ecuyer et al. 2006, 2007). The Fixed Assignment splitting Step IV is specified in Algorithm 4 below. 


\section{Algorithm 4. Fixed assignment splitting in IPS step IV}

IV. Splitting: $\left\{\tilde{\xi}_{k}^{j}\right\}_{j=1}^{N_{S_{k}}}$ is a random permutations of $\left\{\widetilde{\xi}_{k}^{j}\right\}_{j=1}^{N_{S_{k}}}$.

Copy: $\quad \xi_{k}^{i}=\widetilde{\xi}_{k}^{i}$ for $i=1, \ldots, N_{S_{k}}$;

$$
\begin{aligned}
& \xi_{k}^{i+N_{S_{k}}}=\widetilde{\xi}_{k}^{i} \text { for } i=1, \ldots, N_{S_{k}} ; \\
& \ldots \\
& \xi_{k}^{i+\left\lfloor\frac{N_{p}}{N_{S_{k}}}-1\right\rfloor^{N_{S_{k}}}}=\widetilde{\xi}_{k}^{i} \text { for } i=1, \ldots, N_{S_{k}} ; \\
& \xi_{k}^{i+\left\lfloor\frac{N_{p}}{N_{S_{k}}}\right\rfloor^{N_{S_{k}}}}=\widetilde{\xi}_{k}^{i} \text { for } i=1, \ldots, N_{p}-\left\lfloor\frac{N_{p}}{N_{S_{k}}}\right\rfloor N_{S_{k}} .
\end{aligned}
$$

Each particle receives weight $1 / N_{p}$

Straightforward verification shows that Proposition 1 and Theorem 1 also hold true using fixed assignment splitting in IPS step IV.

Proposition 5: If $N_{S_{k}} \geq 2$, and we use fixed assignment splitting at IPS level $k$ then.

$$
\begin{gathered}
\mathbb{E}\left\{\bar{\gamma}_{k+1} \mid \tilde{\xi}_{k}^{1}, \ldots, \tilde{\xi}_{k}^{N_{S_{k}}}\right\}=\frac{1}{N_{S_{k}}} \sum_{i=1}^{N_{S_{k}}} \gamma_{k+1}\left(\tilde{\xi}_{k}^{i}\right) \\
\operatorname{Var}\left\{\bar{\gamma}_{k+1} \mid \tilde{\xi}_{k}^{1}, \ldots, \tilde{\xi}_{k}^{N_{S_{k}}}\right\} \\
=\frac{1}{N_{p} N_{S_{k}}} \sum_{i=1}^{N_{S_{k}}}\left[\gamma_{k+1}\left(\tilde{\xi}_{k}^{i}\right)\left(1-\gamma_{k+1}\left(\tilde{\xi}_{k}^{i}\right)\right)\right] \\
+\frac{\left(N_{p} \bmod N_{S_{k}}\right)\left[N_{S_{k}}-\left(N_{p} \bmod N_{S_{k}}\right)\right]}{N_{p}^{2} N_{S_{k}}\left(N_{S_{k}}-1\right)} \\
{\left[\sum_{i=1}^{N_{S_{k}}}\left[\left(\gamma_{k+1}\left(\tilde{\xi}_{k}^{i}\right)\right)^{2}\right]-\frac{1}{N_{S_{k}}} \sum_{i=1}^{N_{S_{k}}} \sum_{i^{\prime}=1}^{N_{S_{k}}}\left[\gamma_{k+1}\left(\tilde{\xi}_{k}^{i}\right) \gamma_{k+1}\left(\tilde{\xi}_{k}^{i^{\prime}}\right)\right]\right.}
\end{gathered}
$$

with mod representing modulo operation.

Proof: See 8 .. 


\section{Comparison of Variances}

This section proves that IPS using fixed assignment splitting has variance advantage over IPS under each of the three Random assignment splitting methods MR, MS and RMS. This is accomplished through a sequence of three Theorems. Theorem 2 compares the four splitting strategies at a single level only. Theorem 3 considers multiple levels, with difference in splitting strategies at a single level and no differences in splitting strategy at the other levels. Theorem 4 uses Theorem 3 to complete the comparison of IPS under different ways of splitting.

Theorem 2: Given successful particles $\tilde{\xi}_{k}^{1}, \ldots, \tilde{\xi}_{k}^{N_{S_{k}}}$ at IPS level $k$ with $N_{S_{k}} \geq 1$. The dominance of the four splitting methods (MR, MS, RMS, FAS) in terms of $\operatorname{Var}\left\{\bar{\gamma}_{k+1} \mid \tilde{\xi}_{k}^{1}, \ldots, \tilde{\xi}_{k}^{N_{S_{k}}}\right\}$ is:

$$
\mathrm{V}_{F A S}^{k} \leq \mathrm{V}_{R M S}^{k} \leq \mathrm{V}_{M S}^{k} \leq \mathrm{V}_{M R}^{k}
$$

Theorem 3: If IPS levels 1 to $k$-1 make use of the same type of splitting (either MR, MS, $R M S$ or FAS), then the dominance of the four splitting methods at level $k$, in terms of $\operatorname{Var}\left\{\prod_{k^{\prime}=1}^{k} \bar{\gamma}_{k^{\prime}}\right\}$ satisfies:

$$
\mathrm{V}_{F A S \_k} \leq \mathrm{V}_{R M S \_k} \leq \mathrm{V}_{M S \_k} \leq \mathrm{V}_{M R \_k}
$$

Theorem 4: Under the same type of Splitting (either MR, MS, RMS or FAS) at all levels, then the dominance of the four splitting methods in terms of $\operatorname{Var}\{\bar{\gamma}\}$ satisfies:

$$
\mathrm{V}_{F A S} \leq \mathrm{V}_{R M S} \leq \mathrm{V}_{M S} \leq \mathrm{V}_{M R}
$$

Proof of Theorem 2: From inequality of arithmetic and geometric means we know:

$$
\sum_{i=1}^{N_{S_{k}}}\left(\gamma_{k+1}\left(\tilde{\xi}_{k}^{i}\right)\right)^{2} \geq \frac{1}{N_{S_{k}}}\left(\sum_{i=1}^{N_{S_{k}}} \gamma_{k+1}\left(\tilde{\xi}_{k}^{i}\right)\right)^{2}
$$

The right hand term equals:

$$
\left(\sum_{i=1}^{N_{S_{k}}} \gamma_{k+1}\left(\tilde{\xi}_{k}^{i}\right)\right)^{2}=\sum_{i=1}^{N_{S_{k}}} \sum_{i^{\prime}=1}^{N_{S_{k}}} \gamma_{k+1}\left(\tilde{\xi}_{k}^{i}\right) \gamma_{k+1}\left(\tilde{\xi}_{k}^{i^{\prime}}\right)
$$

Substituting this in (25) yields:

$$
\left[\sum_{i=1}^{N_{S_{k}}}\left[\left(\gamma_{k+1}\left(\tilde{\xi}_{k}^{i}\right)\right)^{2}\right]-\frac{1}{N_{S_{k}}} \sum_{i=1}^{N_{S_{k}}} \sum_{i^{\prime}=1}^{N_{S_{k}}}\left[\gamma_{k+1}\left(\tilde{\xi}_{k}^{i}\right) \gamma_{k+1}\left(\tilde{\xi}_{k}^{i^{\prime}}\right)\right]\right] \geq 0
$$

If $N_{S_{k}}=1$, then all four splitting methods do the same. If $N_{S_{k}} \geq 2$, we have to compare variances in Propositions 2, 3, 4 and 5. Due to (27) for $\operatorname{Var}_{M S} \leq \operatorname{Var}_{M R}, \operatorname{Var}_{R M S} \leq \operatorname{Var}_{M S}$ and $\operatorname{Var}_{F A S} \leq \operatorname{Var}_{R M S}$ this means we have to verify: 


$$
\begin{gathered}
\frac{\left(N_{p}-N_{S_{k}}\right)}{N_{p} N_{S_{k}}} \leq \frac{1}{N_{S_{k}}} \\
\left(N_{p} \bmod N_{S_{k}}\right) \leq\left(N_{p}-N_{S_{k}}\right) \\
\frac{\left(N_{p} \bmod N_{S_{k}}\right)\left[N_{S_{k}}-\left(N_{p} \bmod N_{S_{k}}\right)\right]}{\left(N_{S_{k}}-1\right)} \leq\left(N_{p} \bmod N_{S_{k}}\right)
\end{gathered}
$$

From $\frac{1}{N_{S_{k}}}-\frac{1}{N_{p}} \leq \frac{1}{N_{S_{k}}}$ follows that inequality (28) holds true. Substituting $\alpha_{k} N_{S_{k}}+$ $\left(N_{p} \bmod N_{S_{k}}\right)=N_{p}$ in. $\left(N_{p} \bmod N_{S_{k}}\right) \leq\left(N_{p} \bmod N_{S_{k}}\right)+N_{S_{k}}\left(\alpha_{k}-1\right)$ and subsequent rearrangement of terms proves (29).

Because $\left(N_{p} \bmod N_{S_{k}}\right)^{2} \geq\left(N_{p} \bmod N_{S_{k}}\right)$ we get:

$$
\left(N_{p} \bmod N_{S_{k}}\right)\left[N_{S_{k}}-\left(N_{p} \bmod N_{S_{k}}\right)\right] \leq\left(N_{p} \bmod N_{S_{k}}\right)\left(N_{S_{k}}-1\right)
$$

Dividing both sides by $\left(N_{S_{k}}-1\right)$ proves (30). Q.E.D.

Proof of Theorem 3: By defining the notation $\bar{\gamma}_{k}^{\pi} \triangleq \prod_{k^{\prime}=1}^{k} \bar{\gamma}_{k^{\prime}}$, we can write $\bar{\gamma}_{k}^{\pi}=\bar{\gamma}_{k} \bar{\gamma}_{k-1}^{\pi}$. By also defining the sigma algebra $C_{\tilde{\xi}}^{k}=\sigma\left\{\tilde{\xi}_{k^{\prime}}^{i} ; i=1, \ldots, N_{S_{k^{\prime}}}, k^{\prime}=1, \ldots, k\right\}$, we can subsequently derive:

$$
\mathbb{E}\left\{\bar{\gamma}_{k-1}^{\pi} \bar{\gamma}_{k} \mid C_{\tilde{\xi}}^{k-1}\right\} \stackrel{a}{=} \bar{\gamma}_{k-1}^{\pi} \mathbb{E}\left\{\bar{\gamma}_{k} \mid C_{\tilde{\xi}}^{k-1}\right\}
$$

where equality (a) holds because $C_{\tilde{\xi}}^{k-1} \subset \sigma\left\{\bar{\gamma}_{k-1}^{\pi}\right\}$.

If $N_{S_{k}}=0$, then we have $\bar{\gamma}_{k}=\frac{N_{S_{k}}}{N_{p}}=0$ and

$$
\operatorname{Var}\left\{\bar{\gamma}_{k-1}^{\pi} \bar{\gamma}_{k}\right\}=\operatorname{Var}\left\{\bar{\gamma}_{k-1}^{\pi} \cdot 0\right\}=0
$$

Thus if $N_{S_{k}}=0$, then $\operatorname{Var}\left\{\bar{\gamma}_{k}^{\pi}\right\}$ is the same under any of the four splitting methods. If $N_{S_{k}} \geq 1$, we can derive as follows by using the law of total variance:

$$
\begin{aligned}
& \operatorname{Var}\left\{\bar{\gamma}_{k}^{\pi}\right\}=\operatorname{Var}\left\{\bar{\gamma}_{k-1}^{\pi} \bar{\gamma}_{k}\right\} \\
& =\mathbb{E}\left\{\operatorname{Var}\left\{\bar{\gamma}_{k-1}^{\pi} \bar{\gamma}_{k} \mid C_{\tilde{\xi}}^{k-1}\right\}\right\}+\operatorname{Var}\left\{\mathbb{E}\left\{\bar{\gamma}_{k-1}^{\pi} \bar{\gamma}_{k} \mid C_{\tilde{\xi}}^{k-1}\right\}\right\} \\
& =\mathbb{E}\left\{\left(\bar{\gamma}_{k-1}^{\pi}\right)^{2} \operatorname{Var}\left\{\bar{\gamma}_{k} \mid C_{\tilde{\xi}}^{k-1}\right\}\right\}+\operatorname{Var}\left\{\mathbb{E}\left\{\bar{\gamma}_{k-1}^{\pi} \bar{\gamma}_{k} \mid C_{\tilde{\xi}}^{k-1}\right\}\right\}
\end{aligned}
$$

By using $\operatorname{Var}\{X\}=\mathbb{E}\left\{X^{2}\right\}+\{\mathbb{E}(X)\}^{2},(33)$ becomes:

$$
\begin{aligned}
& \operatorname{Var}\left\{\bar{\gamma}_{k}^{\pi}\right\}=\mathbb{E}\left\{\left(\bar{\gamma}_{k-1}^{\pi}\right)^{2} \operatorname{Var}\left\{\bar{\gamma}_{k} \mid C_{\tilde{\xi}}^{k-1}\right\}\right\} \\
& +\mathbb{E}\left\{\left[\mathbb{E}\left\{\bar{\gamma}_{k-1}^{\pi} \bar{\gamma}_{k} \mid C_{\tilde{\xi}}^{k-1}\right\}\right]^{2}\right\}-\left\{\mathbb{E}\left[\mathbb{E}\left(\bar{\gamma}_{k-1}^{\pi} \bar{\gamma}_{k} \mid C_{\tilde{\xi}}^{k-1}\right)\right]\right\}^{2}
\end{aligned}
$$

Using the property of the conditional expectation, we can derive: 


$$
\begin{gathered}
\operatorname{Var}\left\{\bar{\gamma}_{k}^{\pi}\right\}=\mathbb{E}\left\{\left(\bar{\gamma}_{k-1}^{\pi}\right)^{2} \operatorname{Var}\left\{\bar{\gamma}_{k} \mid C_{\tilde{\xi}}^{k-1}\right\}\right\} \\
+\mathbb{E}\left\{\left[\bar{\gamma}_{k-1}^{\pi} \mathbb{E}\left\{\bar{\gamma}_{k} \mid C_{\tilde{\xi}}^{k-1}\right\}\right]^{2}\right\}-\left\{\mathbb{E}\left(\bar{\gamma}_{k-1}^{\pi} \bar{\gamma}_{k}\right)\right\}^{2}
\end{gathered}
$$

Further evaluation of (35) yields:

$$
\begin{gathered}
\operatorname{Var}\left\{\bar{\gamma}_{k}^{\pi}\right\} \\
=\mathbb{E}\left\{\left(\bar{\gamma}_{k-1}^{\pi}\right)^{2} \operatorname{Var}\left\{\bar{\gamma}_{k} \mid C_{\tilde{\xi}}^{k-1}\right\}\right\} \\
+\mathbb{E}\left\{\left(\bar{\gamma}_{k-1}^{\pi}\right)^{2}\left[\mathbb{E}\left\{\bar{\gamma}_{k} \mid C_{\tilde{\xi}}^{k-1}\right\}\right]^{2}\right\}-\left\{\gamma_{k}^{\pi}\right\}^{2} \\
=\mathbb{E}\left\{\left(\bar{\gamma}_{k-1}^{\pi}\right)^{2} \operatorname{Var}\left\{\bar{\gamma}_{k} \mid C_{\tilde{\xi}}^{k-1}\right\}+\left(\bar{\gamma}_{k-1}^{\pi}\right)^{2}\left[\mathbb{E}\left\{\bar{\gamma}_{k} \mid C_{\tilde{\xi}}^{k-1}\right\}\right]^{2}\right\} \\
-\left\{\gamma_{k}^{\pi}\right\}^{2} \\
=\mathbb{E}\left\{\left(\bar{\gamma}_{k-1}^{\pi}\right)^{2} \operatorname{Var}\left\{\bar{\gamma}_{k} \mid C_{\tilde{\xi}}^{k-1}\right\}\right\} \\
+\mathbb{E}\left\{\left(\bar{\gamma}_{k-1}^{\pi}\right)^{2}\left[\mathbb{E}\left\{\bar{\gamma}_{k} \mid C_{\tilde{\xi}}^{k-1}\right\}\right]^{2}\right\}-\left\{\gamma_{k}^{\pi}\right\}^{2} \\
+\int p_{\bar{\gamma}_{k-1}^{\pi}}(y) y^{2} \mathbb{E}\left\{\operatorname{Var}\left\{\bar{\gamma}_{k} \mid C_{\tilde{\xi}}^{k-1}\right\} \mid \bar{\gamma}^{k-1}=y\right\} d y \\
y_{k-1}^{2} \mathbb{E}\left\{\left[\mathbb{E}\left\{\bar{\gamma}_{k} \mid C_{\tilde{\xi}}^{k-1}\right\}\right]^{2} \mid \bar{\gamma}^{k-1}=y\right\} d y-\left\{\gamma_{k}^{\pi}\right\}^{2}
\end{gathered}
$$

To complete the proof we have to compare (36) under the four splitting methods. $\mathbb{E}\left\{\bar{\gamma}_{k+1} \mid C_{\tilde{\xi}}^{k}\right\}$ equals under each of the four splitting methods. To compare the variance term we denote by $\mathrm{V}_{F A S}^{k}, \mathrm{~V}_{R M S}^{k}, \mathrm{~V}_{M S}^{k}$ and $\mathrm{V}_{M R}^{k}$ the $\operatorname{Var}\left\{\bar{\gamma}_{k} \mid C_{\tilde{\xi}}^{k-1}\right\}$ under FAS, RMS, MS and MR respectively. From Theorem 2, we know $\mathrm{V}_{F A S}^{k} \leq \mathrm{V}_{R M S}^{k} \leq \mathrm{V}_{M S}^{k} \leq \mathrm{V}_{M R}^{k}$ at level $k$.

Due to the monotonicity of conditional expectation, this implies $\mathbb{E}\left\{\mathrm{V}_{F A S}^{k} \bar{\gamma} \frac{\pi}{k-1}=y\right\} \leq$ $\mathbb{E}\left\{\mathrm{V}_{R M S}^{k} \mid \bar{\gamma}_{k-1}^{\pi}=y\right\} \leq \mathbb{E}\left\{\mathrm{V}_{M S}^{k} \mid \bar{\gamma}_{k-1}^{\pi}=y\right\} \leq \mathbb{E}\left\{\mathrm{V}_{R M S}^{k} \mid \bar{\gamma}_{k-1}^{\pi}=y\right\}$. For (36) this means that if $N_{S_{k}} \geq 1$, then $\operatorname{Var}\left\{\bar{\gamma}_{k}^{\pi}\right\}$ under FAS, RMS, MS and MR satisfy inequality (24). Q.E.D.

Proof of Theorem 4: Theorem 3 shows that it is advantageous to use FAS at level $k$, whatever splitting types are used at level 1 to level $k-1$. For $k=m$, this implies an advantage to use FAS at level $m$. The same reasoning shows that it also is advantageous to use FAS at level $k=m-1$. This reasoning can be repeated for level $m-2, m-3, \ldots, k=2$. At level $k=1$, there is no difference between the two splitting strategies. Therefore, we can conclude that if all levels make use of FAS, then $\operatorname{Var}\{\bar{\gamma}\}$ is less than or equal to that when all levels make use of RMS, i.e. $\mathrm{V}_{F A S} \leq \mathrm{V}_{R M S}$. This reasoning is also be applied for RMS relative to MS and MR, which yields $\mathrm{V}_{R M S} \leq \mathrm{V}_{M S}$. Finally this reasoning is applied to MS relative to MR, which yields $\mathrm{V}_{M S} \leq \mathrm{V}_{M R}$. Q.E.D. 


\section{Simulation Example}

\subsection{Geometric Brownian Motion Example}

Following Krystul (2006, pp. 22-26) in this section we apply IPS for the estimation reach probability for a Geometric Brownian motion, and compare the results under Fixed Assignment splitting versus those under multinomial splitting versus those under multinomial resampling. The SDE of Geometric Brownian motion satisfies:

$$
d X_{t}=\left(\mu+\frac{\sigma^{2}}{2}\right) X_{t} d t+\sigma X_{t} d W_{t}
$$

where $\mu>0, \sigma>0$ and $X_{0} \geq 1$. We want to estimate the probability $\mathbb{P} \tau<T$ with $\tau \triangleq \inf \left\{t>0: X_{t} \geq L\right\}$.

\subsection{Analytical and MC Simulation Results}

Thanks to (Tuckwell and Wan 1984; Karlin and Taylor 1975, p363, Theorem 5.3), we can use the following equation to evaluate reach probabilities:

$$
\gamma=\mathbb{P}(\tau<T)=\int_{0}^{T} \frac{\ln \left(L / X_{0}\right)}{\sqrt{2 \pi \sigma^{2} t^{3}}} \exp \left\{\frac{-\left(\ln \left(L / X_{0}\right)-\mu t\right)^{2}}{2 \sigma^{2} t}\right\} d t
$$

For this example, we use (38) to set the levels $\left\{L_{k}, k=1, \ldots, m\right\}$, such that the conditional probabilities between successive levels are equal to $1 / 10$. Table 1 shows the resulting $L_{k}$ level values for $k=1,2, \ldots$, as well as the analytical $\gamma_{k}$ and $\gamma$ results for these levels. The right columns in Table 1 also show the $\bar{\gamma}_{M C}$ results obtained through straightforward Monte Carlo (MC) simulation using 10,000 runs with numerical integration time step $\Delta=2 \times 10^{-3} s$. The results in Table 1 show that straightforward MC simulation based estimation of $\gamma$ fails to work beyond $k=4$. Instead of stopping the simulation of the $i$-th particle at each stopping times $\tau_{k}^{i}$, we stop it at $t_{k}^{i}$, i.e. the end of the first integration time step that $x_{t_{k}^{i}}^{i}$ is at or has passed level $k$. The implication is that it remains to be verified if the numerical time step $\Delta$ of the IPS simulation is small enough.

Table 1 Analytical and MC estimated $\gamma$ and $\gamma_{k}, k=1 . .10$, for geometric Brownian motion example, with $\mu=1, \sigma=1$, $X_{0}=1, T=1 s$ and $L=1717.25$. The MC estimated $\bar{\gamma}_{M C}$ used 10,000 runs with $\Delta=2 \times 10^{-3} s$

\begin{tabular}{lllll}
\hline$k$ & $L_{k}$ & $\gamma_{k}$ & $\gamma$ & $\bar{\gamma}_{M C}$ \\
\hline 1 & 12.27 & 0.09998 & 0.09998 & 0.0957 \\
2 & 33.038 & $1.000 \times 10^{-1}$ & $1.000 \times 10^{-2}$ & 0.0085 \\
3 & 69.09 & $1.000 \times 10^{-1}$ & $1.000 \times 10^{-3}$ & $6.000 \times 10^{-4}$ \\
4 & 127.45 & $1.001 \times 10^{-1}$ & $1.001 \times 10^{-4}$ & $1.000 \times 10^{-4}$ \\
5 & 217.5 & $1.000 \times 10^{-1}$ & $1.000 \times 10^{-5}$ & 0 \\
6 & 351.445 & $1.000 \times 10^{-1}$ & $1.000 \times 10^{-6}$ & 0 \\
7 & 545.14 & $1.000 \times 10^{-1}$ & $1.000 \times 10^{-7}$ & 0 \\
8 & 818.935 & $1.000 \times 10^{-1}$ & $1.000 \times 10^{-8}$ & 0 \\
9 & 1198.75 & $1.000 \times 10^{-1}$ & $1.000 \times 10^{-9}$ & 0 \\
10 & 1717.25 & $1.000 \times 10^{-1}$ & $1.000 \times 10^{-10}$ & 0 \\
\hline
\end{tabular}


Table 2 Multiple times IPS simulation results under Multinomial Resampling vs. Multinomial splitting vs. Fixed Assignment splitting for the setting of Table $1, \Delta=2 \times 10^{-3} s, N_{p}=1000$ and $N_{I P S}=1000$

\begin{tabular}{|c|c|c|c|c|c|c|c|c|c|}
\hline \multirow[b]{2}{*}{$k$} & \multicolumn{3}{|c|}{ Multinomial Resampling } & \multicolumn{3}{|c|}{ Multinomial splitting } & \multicolumn{3}{|c|}{ Fixed Assignment splitting } \\
\hline & $\hat{\gamma}$ & $\rho_{S}$ & $\hat{c}_{\hat{\gamma}, N R M S E}$ & $\hat{\gamma}$ & $\rho_{S}$ & $\hat{c}_{\hat{\gamma}, N R M S E}$ & $\hat{\gamma}$ & $\rho_{S}$ & $\hat{c}_{\hat{\gamma}, N R M S E}$ \\
\hline 1 & $9.51 \times 10^{-2}$ & $100 \%$ & $11 \%$ & $9.60 \times 10^{-2}$ & $100 \%$ & $10 \%$ & $9.51 \times 10^{-2}$ & $100 \%$ & $11 \%$ \\
\hline 2 & $9.29 \times 10^{-3}$ & $100 \%$ & $19 \%$ & $9.33 \times 10^{-3}$ & $100 \%$ & $19 \%$ & $9.31 \times 10^{-3}$ & $100 \%$ & $18 \%$ \\
\hline 3 & $9.17 \times 10^{-4}$ & $100 \%$ & $30 \%$ & $9.21 \times 10^{-4}$ & $100 \%$ & $29 \%$ & $9.11 \times 10^{-4}$ & $100 \%$ & $28 \%$ \\
\hline 4 & $9.10 \times 10^{-5}$ & $100 \%$ & $46 \%$ & $9.11 \times 10^{-5}$ & $100 \%$ & $46 \%$ & $9.04 \times 10^{-5}$ & $100 \%$ & $43 \%$ \\
\hline 5 & $8.96 \times 10^{-6}$ & $100 \%$ & $71 \%$ & $8.95 \times 10^{-6}$ & $100 \%$ & $69 \%$ & $8.89 \times 10^{-6}$ & $100 \%$ & $65 \%$ \\
\hline 6 & $8.90 \times 10^{-7}$ & $100 \%$ & $110 \%$ & $8.85 \times 10^{-7}$ & $100 \%$ & $102 \%$ & $8.67 \times 10^{-7}$ & $100 \%$ & $95 \%$ \\
\hline 7 & $9.07 \times 10^{-8}$ & $99 \%$ & $176 \%$ & $8.67 \times 10^{-8}$ & $99 \%$ & $158 \%$ & $8.42 \times 10^{-8}$ & $100 \%$ & $134 \%$ \\
\hline 8 & $9.31 \times 10^{-9}$ & $96 \%$ & $292 \%$ & $8.60 \times 10^{-9}$ & $95 \%$ & $253 \%$ & $8.06 \times 10^{-9}$ & $97 \%$ & $182 \%$ \\
\hline 9 & $9.72 \times 10^{-10}$ & $86 \%$ & $488 \%$ & $8.59 \times 10^{-10}$ & $88 \%$ & $398 \%$ & $7.81 \times 10^{-10}$ & $88 \%$ & $243 \%$ \\
\hline 10 & $1.10 \times 10^{-10}$ & $69 \%$ & $912 \%$ & $8.36 \times 10^{-11}$ & $73 \%$ & $559 \%$ & $7.29 \times 10^{-11}$ & $73 \%$ & $292 \%$ \\
\hline
\end{tabular}

Table 3 Multiple times IPS simulation results under Multinomial Resampling vs. Multinomial splitting vs. Fixed Assignment splitting for the setting of Table $1, \Delta=4 \times 10^{-4} s, N_{p}=1000$ and $N_{I P S}=1000$

\begin{tabular}{|c|c|c|c|c|c|c|c|c|c|}
\hline \multirow[b]{2}{*}{$k$} & \multicolumn{3}{|c|}{ Multinomial Resampling } & \multicolumn{3}{|c|}{ Multinomial splitting } & \multicolumn{3}{|c|}{ Fixed Assignment splitting } \\
\hline & $\hat{\gamma}$ & $\rho_{S}$ & $\hat{c}_{\hat{\gamma}, N R M S E}$ & $\hat{\gamma}$ & $\rho_{S}$ & $\hat{c}_{\hat{\gamma}, N R M S E}$ & $\hat{\gamma}$ & $\rho_{S}$ & $\hat{c}_{\hat{\gamma}, N R M S E}$ \\
\hline 1 & $9.81 \times 10^{-2}$ & $100 \%$ & $10 \%$ & $9.77 \times 10^{-2}$ & $100 \%$ & $10 \%$ & $9.78 \times 10^{-2}$ & $100 \%$ & $10 \%$ \\
\hline 2 & $9.75 \times 10^{-3}$ & $100 \%$ & $18 \%$ & $9.73 \times 10^{-3}$ & $100 \%$ & $17 \%$ & $9.70 \times 10^{-3}$ & $100 \%$ & $17 \%$ \\
\hline 3 & $9.63 \times 10^{-4}$ & $100 \%$ & $29 \%$ & $9.72 \times 10^{-4}$ & $100 \%$ & $29 \%$ & $9.58 \times 10^{-4}$ & $100 \%$ & $28 \%$ \\
\hline 4 & $9.49 \times 10^{-5}$ & $100 \%$ & $46 \%$ & $9.76 \times 10^{-5}$ & $100 \%$ & $46 \%$ & $9.43 \times 10^{-5}$ & $100 \%$ & $44 \%$ \\
\hline 5 & $9.47 \times 10^{-6}$ & $100 \%$ & $74 \%$ & $9.81 \times 10^{-6}$ & $100 \%$ & $73 \%$ & $9.18 \times 10^{-6}$ & $100 \%$ & $66 \%$ \\
\hline 6 & $9.56 \times 10^{-7}$ & $100 \%$ & $114 \%$ & $9.95 \times 10^{-7}$ & $100 \%$ & $112 \%$ & $8.98 \times 10^{-7}$ & $100 \%$ & $94 \%$ \\
\hline 7 & $9.86 \times 10^{-8}$ & $99 \%$ & $173 \%$ & $1.00 \times 10^{-7}$ & $99 \%$ & $169 \%$ & $8.65 \times 10^{-8}$ & $100 \%$ & $131 \%$ \\
\hline 8 & $1.03 \times 10^{-8}$ & $95 \%$ & $261 \%$ & $1.02 \times 10^{-8}$ & $96 \%$ & $241 \%$ & $8.23 \times 10^{-9}$ & $96 \%$ & $179 \%$ \\
\hline 9 & $1.09 \times 10^{-9}$ & $86 \%$ & $390 \%$ & $1.04 \times 10^{-9}$ & $89 \%$ & $376 \%$ & $7.68 \times 10^{-10}$ & $89 \%$ & $235 \%$ \\
\hline 10 & $1.14 \times 10^{-10}$ & $72 \%$ & $554 \%$ & $1.02 \times 10^{-10}$ & $74 \%$ & $498 \%$ & $7.17 \times 10^{-11}$ & $75 \%$ & $301 \%$ \\
\hline
\end{tabular}

\subsection{IPS Simulation Results}

In this subsection we apply IPS under Multinomial resampling, under multinomial splitting and under fixed assignment splitting. By repeating IPS $N_{I P S}$ times estimates of the rate of surviving IPS, $\rho_{S}$, and Normalized root-mean-square error, $\hat{c}_{\hat{\gamma}, N R M S E}$. The results are shown in Table 2 with $\Delta=2 \times 10^{-3} s$ and Table 3 with $\Delta=4 \times 10^{-4} s$, for $N_{p}=1000$ and $N_{I P S}=1000$. The measures $\hat{\gamma}, \rho_{S}$ and $\hat{c}_{\hat{\gamma}, N R M S E}$ are defined as follows:

$$
\hat{\gamma}=\frac{\sum_{i=1}^{N_{I P S}} \bar{\gamma}^{i}}{N_{I P S}}
$$




$$
\begin{array}{r}
\rho_{S}=\frac{\sum_{i=1}^{N_{I P S}} 1_{\bar{\gamma}^{i}>0}}{N_{I P S}} \\
\text { with } 1_{\bar{\gamma}^{i}>0}=\left\{\begin{array}{l}
1, \text { if } \bar{\gamma}^{i}>0 \\
0, \text { if } \bar{\gamma}^{i}=0
\end{array} \text { and } \hat{c}_{\hat{\gamma}, N R M S E}=\frac{R M S E}{\gamma} \times 100 \%\right.
\end{array}
$$

where $\bar{\gamma}^{i}$ denotes the estimated reach probability for the $i$-th IPS simulation.

The results in Tables 2 and 3 show that the Normalized Root-Mean-Square Error, $\hat{c}_{\hat{\gamma}, N R M S E}$, is under Fixed Assignment splitting better than under Multinomial splitting which is better than under Multinomial Resampling. This difference in $\hat{c}_{\hat{\gamma}, N R M S E}$ increases with $k$.

\section{Conclusion}

This paper has studied the estimation of the reach probability of an unsafe set by a multidimensional diffusion process using the Interacting Particle System (IPS) framework of Cérou et al. (2006). More specifically it has been proven that IPS using fixed assignment splitting (FAS) dominates in variance reduction over IPS using multinomial resampling (MR), multinomial splitting (MS), and residual multinomial splitting (RMS).

First, in Sect. 3, a novel characterization has been derived for the conditional variance at level $\mathrm{k}$ in Theorem 1. This has been elaborated in Propositions 2, 3, 4 and 5 for MR, MS, RMS and FAS respectively. Subsequently, the conditional variances are compared in Sect. 5 through Theorems 2, 3 and 4. Theorem 2 proves the aimed results for an arbitrary single level $k$, given the same set of survived particles at the beginning of this level. Subsequently Theorem 3 proves the aimed results for an arbitrary single level $k$, under the condition that there are no differences in splitting strategy used at all earlier levels. Finally Theorem 4 completes the proof by induction using Theorem 3 .

In Section 5, the difference in IPS performances under different splitting methods has been illustrated for a one-dimensional geometric Brownian motion example for which the reach probabilities are analytically known.

Open Access This article is licensed under a Creative Commons Attribution 4.0 International License, which permits use, sharing, adaptation, distribution and reproduction in any medium or format, as long as you give appropriate credit to the original author(s) and the source, provide a link to the Creative Commons licence, and indicate if changes were made. The images or other third party material in this article are included in the article's Creative Commons licence, unless indicated otherwise in a credit line to the material. If material is not included in the article's Creative Commons licence and your intended use is not permitted by statutory regulation or exceeds the permitted use, you will need to obtain permission directly from the copyright holder. To view a copy of this licence, visit http://creativecommons.org/licenses/by/4.0/. 


\section{Appendix A. Conditional variance derivations}

\section{Proof of Proposition 1}

If we consider the particles $\xi_{k}^{j}$ copies from $\tilde{\xi}_{k}^{i}$ as a group, then for IPS step II in Table 1 at level $k+1, \bar{\gamma}_{k+1}$ can be written as follows:

$$
\bar{\gamma}_{k+1}=\frac{1}{N_{p}} \sum_{i=1}^{N_{p}} 1\left(\bar{\xi}_{k+1}^{i} \in Q_{k+1}\right)=\frac{1}{N_{p}} \sum_{i=1}^{N_{S_{k}}} Y_{k+1}^{k, i}
$$

with $Y_{k+1}^{k, i}$ the number of particles that have reached level $k+1$ after mutation of the $K_{k}^{i}$ copies from $\tilde{\xi}_{k}^{i}$. Hence, $Y_{k+1}^{k, i}$ has a conditional Binomial distribution with size $K_{k}^{i}$ and success probability $\gamma_{k+1}\left(\tilde{\xi}_{k}^{i}\right)$ given $K_{k}^{i}$ and $\tilde{\xi}_{k}^{i}$. Therefore, the pdf of $Y_{k+1}^{k, i}$ can be expressed as (10) and (11). Q.E.D.

\section{Proof of Theorem 1}

Let us define $C_{\tilde{\xi}}^{k}$ and $C_{\tilde{\xi}, K}^{k}$ as follows:

$$
\begin{gathered}
C_{\tilde{\xi}}^{k} \triangleq \sigma\left\{\tilde{\xi}_{k^{\prime}}^{i} ; i=1, \ldots, N_{S_{k^{\prime}}}, k^{\prime}=1, \ldots, k\right\} \\
C_{\tilde{\xi}, K}^{k} \triangleq \sigma\left\{\tilde{\xi}_{k^{\prime}}^{i}, K_{k^{\prime}}^{i} ; i=1, \ldots, N_{S_{k^{\prime}}}, k^{\prime}=1, \ldots, k ;\right\}
\end{gathered}
$$

Substitution of (43) in $\mathbb{E}\left\{\bar{\gamma}_{k+1} \mid C_{\tilde{\xi}}^{k}\right\}$ and subsequent evaluation yields:

$$
\begin{aligned}
& \mathbb{E}\left\{\bar{\gamma}_{k+1} \mid C_{\tilde{\xi}}^{k}\right\}=\mathbb{E}\left\{\frac{1}{N_{p}} \sum_{i=1}^{N_{S_{k}}} Y_{k+1}^{k, i} \mid C_{\tilde{\xi}}^{k}\right\} \\
& =\frac{1}{N_{p}} \sum_{i=1}^{N_{S_{k}}} \mathbb{E}\left\{Y_{k+1}^{k, i} \mid C_{\tilde{\xi}}^{k}\right\} \stackrel{a}{=} \frac{1}{N_{p}} \sum_{i=1}^{N_{S_{k}}} \mathbb{E}\left\{\mathbb{E}\left\{Y_{k+1}^{k, i} \mid C_{\tilde{\xi}, K}^{k}\right\} \mid C_{\tilde{\xi}}^{k}\right\}
\end{aligned}
$$

where equality (a) holds because $C_{\tilde{\xi}, K}^{k} \supset C_{\tilde{\xi}}^{k}$ and $\mathbb{E}\{X \mid Y\}=\mathbb{E}\{\mathbb{E}\{X \mid Y, Z\} \mid Y\}$.

In a similar way, we can derive: 


$$
\begin{aligned}
& \operatorname{Var}\left\{\bar{\gamma}_{k+1} \mid C_{\tilde{\xi}}^{k}\right\}=\operatorname{Var}\left\{\frac{1}{N_{p}} \sum_{i=1}^{N_{S_{k}}} Y_{k+1}^{k, i} \mid C_{\tilde{\xi}}^{k}\right\} \\
& =\frac{1}{N_{p}^{2}} \sum_{i=1}^{N_{S_{k}}} \operatorname{Var}\left\{Y_{k+1}^{k, i} \mid C_{\tilde{\xi}}^{k}\right\}+\frac{1}{N_{p}^{2}} \sum_{i=1}^{N_{S_{k}}} \sum_{\substack{i^{\prime}=1 \\
i^{\prime} \neq i}}^{N_{S_{k}}} \operatorname{Cov}\left\{Y_{k+1}^{k, i}, Y_{k+1}^{k, i^{\prime}} \mid C_{\tilde{\xi}}^{k}\right\} \\
& \stackrel{a}{=} \frac{1}{N_{p}^{2}} \sum_{i=1}^{N_{S_{k}}}\left[\mathbb{E}\left\{\operatorname{Var}\left\{Y_{k+1}^{k, i} \mid C_{\tilde{\xi}, K}^{k}\right\} \mid C_{\tilde{\xi}}^{k}\right\}+\operatorname{Var}\left\{\mathbb{E}\left\{Y_{k+1}^{k, i} \mid C_{\tilde{\xi}, K}^{k}\right\} \mid C_{\tilde{\xi}}^{k}\right\}\right] \\
& +\frac{1}{N_{p}^{2}} \sum_{i=1}^{N_{S_{k}}} \sum_{\substack{i^{\prime}=1 \\
i^{\prime} \neq i}}^{N_{S_{k}}}\left[\mathbb{E}\left\{\mathbb{E}\left\{Y_{k+1}^{k, i} Y_{k+1}^{k, i^{\prime}} \mid C_{\tilde{\xi}, K}^{k}\right\} \mid C_{\tilde{\xi}}^{k}\right\}\right. \\
& \left.-\mathbb{E}\left\{\mathbb{E}\left\{Y_{k+1}^{k, i} \mid C_{\tilde{\xi}, K}^{k}\right\} \mid C_{\tilde{\xi}}^{k}\right\} \mathbb{E}\left\{\mathbb{E}\left\{Y_{k+1}^{k, i^{\prime}} \mid C_{\tilde{\xi}, K}^{k}\right\} \mid C_{\tilde{\xi}}^{k}\right\}\right]
\end{aligned}
$$

where equality (a) holds because of the law of total conditional variance (Bowsher and Swain 2012).

Further evaluation of (47) yields:

$$
\begin{array}{r}
\operatorname{Var}\left\{\bar{\gamma}_{k+1} \mid C_{\tilde{\xi}}^{k}\right\} \stackrel{a}{=} \frac{1}{N_{p}^{2}} \sum_{i=1}^{N_{S_{k}}}\left[\mathbb{E}\left\{\operatorname{Var}\left\{Y_{k+1}^{k, i} \mid C_{\tilde{\xi}, K}^{k}\right\} \mid C_{\tilde{\xi}}^{k}\right\}\right. \\
\left.+\operatorname{Var}\left\{\mathbb{E}\left\{Y_{k+1}^{k, i} \mid C_{\tilde{\xi}, K}^{k}\right\} \mid C_{\tilde{\xi}}^{k}\right\}\right] \\
+\frac{1}{N_{p}^{2}} \sum_{i=1}^{N_{S_{k}}} \sum_{\substack{i^{\prime}=1 \\
i^{\prime} \neq i}}^{N_{S_{k}}}\left[\mathbb{E}\left\{\mathbb{E}\left\{Y_{k+1}^{k, i} \mid C_{\tilde{\xi}, K}^{k}\right\} \mathbb{E}\left\{Y_{k+1}^{k, i^{\prime}} \mid C_{\tilde{\xi}, K}^{k}\right\} \mid C_{\tilde{\xi}}^{k}\right\}\right. \\
\left.-\mathbb{E}\left\{\mathbb{E}\left\{Y_{k+1}^{k, i} \mid C_{\tilde{\xi}, K}^{k}\right\} \mid C_{\tilde{\xi}}^{k}\right\} \mathbb{E}\left\{\mathbb{E}\left\{Y_{k+1}^{k, i^{\prime}} \mid C_{\tilde{\xi}, K}^{k}\right\} \mid C_{\tilde{\xi}}^{k}\right\}\right]
\end{array}
$$

where equality (a) holds because $Y_{k+1}^{k, i}$ and $Y_{k+1}^{k, i^{\prime}}$ are conditionally independent given $C_{\tilde{\xi}, K}^{k}$.

Since each $Y_{k+1}^{k, i}$ has a conditional Binomial distribution with size $K_{k}^{i}$ and success probability $\gamma_{k+1}\left(\tilde{\xi}_{k}^{i}\right)$. Then, by using Binomial distribution properties, we get:

$$
\begin{aligned}
& \mathbb{E}\left\{Y_{k+1}^{k, i} \mid C_{\tilde{\xi}, K}^{k}\right\}=\mathbb{E}\left\{Y_{k+1}^{k, i} \mid K_{k}^{j}, \tilde{\xi}_{k}^{j}, \text { all } j\right\} \\
& \stackrel{a}{=} \mathbb{E}\left\{Y_{k+1}^{k, i} \mid K_{k}^{i}, \tilde{\xi}_{k}^{i}\right\}=K_{k}^{i} \cdot \gamma_{k+1}\left(\tilde{\xi}_{k}^{i}\right)
\end{aligned}
$$

and

$$
\begin{aligned}
& \operatorname{Var}\left\{Y_{k+1}^{k, i} \mid C_{\tilde{\xi}, K}^{k}\right\}=\operatorname{Var}\left\{Y_{k+1}^{k, i} \mid K_{k}^{j}, \tilde{\xi}_{k}^{j}, \text { all } j\right\} \\
& \stackrel{a}{=} \operatorname{Var}\left\{Y_{k+1}^{k, i} \mid K_{k}^{i}, \tilde{\xi}_{k}^{i}\right\}=K_{k}^{i} \gamma_{k+1}\left(\tilde{\xi}_{k}^{i}\right)\left(1-\gamma_{k+1}\left(\tilde{\xi}_{k}^{i}\right)\right)
\end{aligned}
$$

where equality (a) holds because $Y_{k+1}^{k, i}$ is conditionally dependent of $K_{k}^{i}$ and $\tilde{\xi}_{k}^{i}$, but conditionally independent of $K_{k}^{i^{\prime}}$ and $\tilde{\xi}_{k}^{i^{\prime}}$ for $i^{\prime} \neq i$.

Substituting (49) into (46) and subsequent evaluation yields: 


$$
\begin{gathered}
\mathbb{E}\left\{\bar{\gamma}_{k+1} \mid C_{\tilde{\xi}}^{k}\right\}=\frac{1}{N_{p}} \sum_{i=1}^{N_{S_{k}}} \mathbb{E}\left\{K_{k}^{i} \cdot \gamma_{k+1}\left(\tilde{\xi}_{k}^{i}\right) \mid C_{\tilde{\xi}}^{k}\right\} \\
\stackrel{a}{=} \frac{1}{N_{p}} \sum_{i=1}^{N_{S_{k}}}\left[\mathbb{E}\left\{K_{k}^{i} \mid C_{\tilde{\xi}}^{k}\right\} \gamma_{k+1}\left(\tilde{\xi}_{k}^{i}\right)\right] \\
\stackrel{b}{=} \frac{1}{N_{p}} \sum_{i=1}^{N_{S_{k}}}\left[\mathbb{E}\left\{K_{k}^{i} \mid \tilde{\xi}_{k}^{j}, \text { all } j\right\} \gamma_{k+1}\left(\tilde{\xi}_{k}^{i}\right)\right]
\end{gathered}
$$

where equality (a) holds because $\mathbb{E}\{f(Z) Y \mid Z\}=f(Z) \mathbb{E}\{Y \mid Z\}$; equality (b) holds because of Markov property of $\left\{\tilde{\xi}_{k}^{i}\right\}$.

Similarly, substituting (49) and (50) into (48) and subsequent evaluation yields:

$$
\begin{aligned}
& \operatorname{Var}\left\{\bar{\gamma}_{k+1} \mid C_{\tilde{\xi}}^{k}\right\} \\
& =\frac{1}{N_{p}^{2}} \sum_{i=1}^{N_{S_{k}}}\left[\mathbb{E}\left\{K_{k}^{i} \cdot \gamma_{k+1}\left(\tilde{\xi}_{k}^{i}\right)\left(1-\gamma_{k+1}\left(\tilde{\xi}_{k}^{i}\right)\right) \mid C_{\tilde{\xi}}^{k}\right\}\right. \\
& \left.+\operatorname{Var}\left\{K_{k}^{i} \cdot \gamma_{k+1}\left(\tilde{\xi}_{k}^{i}\right) \mid C_{\tilde{\xi}}^{k}\right\}\right] \\
& +\frac{1}{N_{p}^{2}} \sum_{i=1}^{N_{S_{k}}} \sum_{\substack{i^{\prime}=1 \\
i^{\prime} \neq i}}^{N_{S_{k}}}\left[\mathbb{E}\left\{K_{k}^{i} \cdot \gamma_{k+1}\left(\tilde{\xi}_{k}^{i}\right) K_{k}^{i^{\prime}} \cdot \gamma_{k+1}\left(\tilde{\xi}_{k}^{i^{\prime}}\right) \mid C_{\tilde{\xi}}^{k}\right\}\right. \\
& \left.-\mathbb{E}\left\{K_{k}^{i} \cdot \gamma_{k+1}\left(\tilde{\xi}_{k}^{i}\right) \mid C_{\tilde{\xi}}^{k}\right\} \mathbb{E}\left\{K_{k}^{i^{\prime}} \cdot \gamma_{k+1}\left(\tilde{\xi}_{k}^{i^{\prime}}\right) \mid C_{\tilde{\xi}}^{k}\right\}\right] \\
& \stackrel{a}{=} \frac{1}{N_{p}^{2}} \sum_{i=1}^{N_{S_{k}}}\left[\mathbb{E}\left\{K_{k}^{i} \mid C_{\tilde{\xi}}^{k}\right\} \mathbb{E}\left\{\gamma_{k+1}\left(\tilde{\xi}_{k}^{i}\right)\left(1-\gamma_{k+1}\left(\tilde{\xi}_{k}^{i}\right)\right) \mid C_{\tilde{\xi}}^{k}\right\}\right] \\
& +\frac{1}{N_{p}^{2}} \sum_{i=1}^{N_{S_{k}}}\left[\mathbb{E}\left\{\left(K_{k}^{i}\right)^{2} \mid C_{\tilde{\xi}}^{k}\right\} \mathbb{E}\left\{\left(\gamma_{k+1}\left(\tilde{\xi}_{k}^{i}\right)\right)^{2} \mid C_{\tilde{\xi}}^{k}\right\}\right. \\
& \left.-\mathbb{E}\left\{K_{k}^{i} \mid C_{\tilde{\xi}}^{k}\right\}^{2} \mathbb{E}\left\{\gamma_{k+1}\left(\tilde{\xi}_{k}^{i}\right) \mid C_{\tilde{\xi}}^{k}\right\}^{2}\right] \\
& +\frac{1}{N_{p}^{2}} \sum_{i=1}^{N_{S_{k}}} \sum_{\substack{i^{\prime}=1 \\
i^{\prime} \neq i}}^{N_{S_{k}}}\left[\left(\mathbb{E}\left\{K_{k}^{i} \cdot K_{k}^{i^{\prime}} \mid C_{\tilde{\xi}}^{k}\right\}-\mathbb{E}\left\{K_{k}^{i} \mid C_{\tilde{\xi}}^{k}\right\} \mathbb{E}\left\{K_{k}^{i^{\prime}} \mid C_{\tilde{\xi}}^{k}\right\}\right)\right. \\
& \left.. \mathbb{E}\left\{\gamma_{k+1}\left(\tilde{\xi}_{k}^{i}\right) \gamma_{k+1}\left(\tilde{\xi}_{k}^{i^{\prime}}\right) \mid C_{\tilde{\xi}}^{k}\right\}\right]
\end{aligned}
$$

where equality (a) is thanks to $C_{\tilde{\xi}}^{k}$-conditional independence of $\tilde{\xi}_{k}^{i}$ and $K_{k}^{i}$.

Further evaluation of (52) yields: 


$$
\begin{gathered}
\operatorname{Var}\left\{\bar{\gamma}_{k+1} \mid C_{\tilde{\xi}}^{k}\right\} \\
=\frac{1}{N_{p}^{2}} \sum_{i=1}^{N_{S_{k}}}\left[\mathbb{E}\left\{K_{k}^{i} \mid C_{\tilde{\xi}}^{k}\right\} \gamma_{k+1}\left(\tilde{\xi}_{k}^{i}\right)\left(1-\gamma_{k+1}\left(\tilde{\xi}_{k}^{i}\right)\right)\right] \\
+\frac{1}{N_{p}^{2}} \sum_{i=1}^{N_{S_{k}}}\left[\left(\mathbb{E}\left\{\left(K_{k}^{i}\right)^{2} \mid C_{\tilde{\xi}}^{k}\right\}-\mathbb{E}\left\{K_{k}^{i} \mid C_{\tilde{\xi}}^{k}\right\}^{2}\right)\left(\gamma_{k+1}\left(\tilde{\xi}_{k}^{i}\right)\right)^{2}\right] \\
+\frac{1}{N_{p}^{2}} \sum_{i=1}^{N_{S_{k}}} \sum_{i^{\prime}=1}^{N_{S_{k}}}\left[\left(\mathbb{E}\left\{K_{k}^{i} \cdot K_{k}^{i^{\prime}} \mid C_{\tilde{\xi}}^{k}\right\}-\mathbb{E}\left\{K_{k}^{i} \mid C_{\tilde{\xi}}^{k}\right\} \mathbb{E}\left\{K_{k}^{i^{\prime}} \mid C_{\tilde{\xi}}^{k}\right\}\right)\right. \\
=\frac{1}{N_{p}^{2}} \sum_{i=1}^{N_{S_{k}}}\left[\mathbb{E}\left\{\gamma_{k+1}^{i}\left(\tilde{\xi}_{k}^{i^{\prime}}\right) \gamma_{k+1}^{k}\left(\tilde{\xi}_{\tilde{\xi}}^{i^{\prime}}\right)\right)\right] \\
+\frac{1}{N_{p}^{2}} \sum_{i=1}^{N_{S_{k}}}\left[\operatorname{Var}\left\{K_{k+1}^{i} \mid C_{\tilde{\xi}}^{i}\right)\left(1-\gamma_{k+1}\left(\tilde{\xi}_{k}^{i}\right)\right)\right] \\
\left.+\frac{1}{N_{p}^{2}} \sum_{i=1}^{N_{S_{k}}} \sum_{i_{S_{k}}}^{N^{\prime}=1}\left[\operatorname{Cov}\left\{K_{k+1}^{i}, K_{k}^{i^{\prime}} \mid C_{\tilde{\xi}}^{k}\right\} \cdot\left(\tilde{\xi}_{k}^{i}\right)\right)^{2}\right] \\
i^{\prime} \neq i
\end{gathered}
$$

Due to the strong Markov property of $\left\{\tilde{\xi}_{k}^{i}\right\}$, the $C_{\tilde{\xi}}^{k}$ conditioning in (53) can be replaced by the condition $\left\{\tilde{\xi}_{k}^{j}\right.$, all $\left.j\right\}$. Q.E.D.

\section{Proof of Proposition 2}

For multinomial resampling, the vector $\left(K_{k}^{1}, K_{k}^{2}, \ldots, K_{k}^{N_{S_{k}}}\right)$ follows a multinomial distribution with the number of trials equal to $N_{p}$, which means $K_{k}^{1}+K_{k}^{2}+\ldots+K_{k}^{N_{S_{k}}}=N_{p}$, and with equal success probabilities $\frac{1}{N_{S_{k}}}$. Using multinomial distribution properties, we know for $i=1,2, \ldots, N_{S_{k}}$ :

$$
\begin{gathered}
\mathbb{E}\left\{K_{k}^{i} \mid \tilde{\xi}_{k}^{j}, \text { all } j\right\}=N_{p} \cdot \frac{1}{N_{S_{k}}}=\frac{N_{p}}{N_{S_{k}}} \\
\operatorname{Var}\left\{K_{k}^{i} \mid \tilde{\xi}_{k}^{j}, \text { all } j\right\}=N_{p} \frac{1}{N_{S_{k}}}\left(1-\frac{1}{N_{S_{k}}}\right)=\frac{N_{p}}{N_{S_{k}}}\left(1-\frac{1}{N_{S_{k}}}\right)
\end{gathered}
$$

For $K_{k}^{i}$ and $K_{k}^{i^{\prime}}\left(i \neq i^{\prime}\right)$, we can derive:

$$
\operatorname{Cov}\left\{K_{k}^{i} K_{k}^{i^{\prime}} \mid \tilde{\xi}_{k}^{j}, \text { all } j\right\}=-N_{p} \frac{1}{N_{S_{k}}} \frac{1}{N_{S_{k}}}
$$

Substituting (54) into (51) and substituting (54), (55) and (56) into (53) yield: 


$$
\begin{gathered}
\mathbb{E}\left\{\bar{\gamma}_{k+1} \mid \tilde{\xi}_{k}^{j}, \text { all } j\right\}=\frac{1}{N_{p}} \sum_{i=1}^{N_{S_{k}}}\left[\frac{N_{p}}{N_{S_{k}}} \gamma_{k+1}\left(\tilde{\xi}_{k}^{i}\right)\right] \\
\operatorname{Var}\left\{\bar{\gamma}_{k+1} \mid C_{\tilde{\xi}}^{k}\right\} \\
=\frac{1}{N_{p}^{2}} \sum_{i=1}^{N_{S_{k}}}\left[\frac{N_{p}}{N_{S_{k}}} \gamma_{k+1}\left(\tilde{\xi}_{k}^{i}\right)\left(1-\gamma_{k+1}\left(\tilde{\xi}_{k}^{i}\right)\right)\right] \\
+\frac{1}{N_{p}^{2}} \sum_{i=1}^{N_{S_{k}}}\left[\frac{N_{p}}{N_{S_{k}}}\left(1-\frac{1}{N_{S_{k}}}\right)\left(\gamma_{k+1}\left(\tilde{\xi}_{k}^{i}\right)\right)^{2}\right] \\
+\frac{1}{N_{p}^{2}} \sum_{i=1}^{N_{S_{k}}} \sum_{\substack{i^{\prime} \\
i_{S_{k}}}}^{i^{\prime} \neq 1}\left[\frac{-N_{p}}{N_{S_{k}}^{2}}\left(\gamma_{k+1}\left(\tilde{\xi}_{k}^{i}\right) \gamma_{k+1}\left(\tilde{\xi}_{k}^{i^{\prime}}\right)\right)\right]
\end{gathered}
$$

Elaboration of (57) and (58) yields the equations of Proposition 2. Q.E.D.

\section{Proof of Proposition 3}

For Multinomial Splitting, the vector $\left(K_{k}^{1}-1, K_{k}^{2}-1, \ldots, K_{k}^{N_{S_{k}}}-1\right)$ follows a multinomial distribution with the number of trials equal to $N_{p}-N_{S_{k}}$, which means $\left(K_{k}^{1}-1\right)+\left(K_{k}^{2}-1\right)+\ldots+\left(K_{k}^{N_{S_{k}}}-1\right)=N_{p}-N_{S_{k}}$, and with equal success probabilities $\frac{1}{N_{S_{k}}}$. Using multinomial distribution properties, we know for $i=1,2, \ldots, N_{S_{k}}$ :

$$
\begin{gathered}
\mathbb{E}\left\{K_{k}^{i}-1 \mid \tilde{\xi}_{k}^{j}, \text { all } j\right\}=\frac{N_{p}-N_{S_{k}}}{N_{S_{k}}} \\
\operatorname{Var}\left\{K_{k}^{i}-1 \mid \tilde{\xi}_{k}^{j}, \text { all } j\right\}=\left(N_{p}-N_{S_{k}}\right) \frac{1}{N_{S_{k}}}\left(1-\frac{1}{N_{S_{k}}}\right) \\
=\frac{N_{p}-N_{S_{k}}}{N_{S_{k}}}\left(1-\frac{1}{N_{S_{k}}}\right)
\end{gathered}
$$

From (59) and (60), we can derive:

$$
\mathbb{E}\left\{K_{k}^{i} \mid \tilde{\xi}_{k}^{j}, \text { all } j\right\}=\frac{N_{p}}{N_{S_{k}}}
$$

and

$$
\operatorname{Var}\left\{K_{k}^{i} \mid \tilde{\xi}_{k}^{j}, \text { all } j\right\}=\frac{N_{p}-N_{S_{k}}}{N_{S_{k}}}\left(1-\frac{1}{N_{S_{k}}}\right)
$$

For $K_{k}^{i}$ and $K_{k}^{i^{\prime}}\left(i \neq i^{\prime}\right)$, we can derive: 


$$
\begin{aligned}
\operatorname{Cov}\left\{K_{k}^{i} K_{k}^{i^{\prime}} \mid \tilde{\xi}_{k}^{j}, \text { all } j\right\} & =\operatorname{Cov}\left\{\left(K_{k}^{i}-1\right)\left(K_{k}^{i^{\prime}}-1\right) \mid \tilde{\xi}_{k}^{j}, \text { all } j\right\} \\
& \stackrel{a}{=} \frac{-\left(N_{p}-N_{S_{k}}\right)}{N_{S_{k}}^{2}}
\end{aligned}
$$

where equality (a) holds because of the multinomial distribution property on the covariance.

Substituting (61) into (51) and substituting (61), (62) and (63) into (53) yield:

$$
\begin{gathered}
\mathbb{E}\left\{\bar{\gamma}_{k+1} \mid \tilde{\xi}_{k}^{j}, \text { all } j\right\}=\frac{1}{N_{p}} \sum_{i=1}^{N_{S_{k}}}\left[\frac{N_{p}}{N_{S_{k}}} \gamma_{k+1}\left(\tilde{\xi}_{k}^{i}\right)\right] \\
\operatorname{Var}\left\{\bar{\gamma}_{k+1} \mid \tilde{\xi}_{k}^{1}, \ldots, \tilde{\xi}_{k}^{N_{S_{k}}}\right\} \\
=\frac{1}{N_{p}^{2}} \sum_{i=1}^{N_{S_{k}}}\left[\frac{N_{p}}{N_{S_{k}}} \gamma_{k+1}\left(\tilde{\xi}_{k}^{i}\right)\left(1-\gamma_{k+1}\left(\tilde{\xi}_{k}^{i}\right)\right)\right] \\
+\frac{1}{N_{p}^{2}} \sum_{i=1}^{N_{S_{k}}}\left[\frac{N_{p}-N_{S_{k}}}{N_{S_{k}}}\left(1-\frac{1}{N_{S_{k}}}\right)\left(\gamma_{k+1}\left(\tilde{\xi}_{k}^{i}\right)\right)^{2}\right] \\
+\frac{1}{N_{p}^{2}} \sum_{i=1}^{N_{S_{k}}} \sum_{\substack{i^{\prime} \\
i_{S_{k}}}}^{i^{\prime} \neq i}\left[\frac{-\left(N_{p}-N_{S_{k}}\right)}{N_{S_{k}}^{2}}\left(\gamma_{k+1}\left(\tilde{\xi}_{k}^{i}\right) \gamma_{k+1}\left(\tilde{\xi}_{k}^{i^{\prime}}\right)\right)\right]
\end{gathered}
$$

Elaboration of (64) and (65) yields the equations of Proposition 3. Q.E.D.

\section{Proof of Proposition 4}

For residual multinomial splitting, the vector $\left(K_{k}^{1}-\alpha_{k}, K_{k}^{2}-\alpha_{k}, \ldots, K_{k}^{N_{S_{k}}}-\alpha_{k}\right)$ follows a multinomial distribution with the number of trials equal to $\left(N_{p} \bmod N_{S_{k}}\right)$, and with equal success probabilities $\frac{1}{N_{S_{k}}}$. Using multinomial distribution properties, we know:

$$
\begin{gathered}
\mathbb{E}\left\{K_{k}^{i}-\alpha_{k} \mid \tilde{\xi}_{k}^{j}, \text { all } j\right\}=\frac{N_{p} \bmod N_{S_{k}}}{N_{S_{k}}} \\
\operatorname{Var}\left\{K_{k}^{i}-\alpha_{k} \mid \tilde{\xi}_{k}^{j}, \text { all } j\right\}=\frac{\left(N_{p} \bmod N_{S_{k}}\right)}{N_{S_{k}}}\left(1-\frac{1}{N_{S_{k}}}\right)
\end{gathered}
$$

From (66) and (67), we can derive:

$$
\mathbb{E}\left\{K_{k}^{i} \mid \tilde{\xi}_{k}^{j}, \text { all } j\right\}=\frac{\left(N_{p} \bmod N_{S_{k}}\right)}{N_{S_{k}}}+\alpha_{k}=\frac{N_{p}}{N_{S_{k}}}
$$

and 


$$
\operatorname{Var}\left\{K_{k}^{i} \mid \tilde{\xi}_{k}^{j}, \text { all } j\right\}=\frac{\left(N_{p} \bmod N_{S_{k}}\right)}{N_{S_{k}}}\left(1-\frac{1}{N_{S_{k}}}\right)
$$

For $K_{k}^{i}$ and $K_{k}^{i^{\prime}}\left(i \neq i^{\prime}\right)$, we can derive:

$$
\operatorname{Cov}\left\{K_{k}^{i} K_{k}^{i^{\prime}} \mid \tilde{\xi}_{k}^{j}, \text { all } j\right\}=-\left(N_{p} \bmod N_{S_{k}}\right) \frac{1}{N_{S_{k}}} \frac{1}{N_{S_{k}}}
$$

Substituting (68) into (51) and substituting (68), (69) and (70) into (53) yield:

$$
\begin{gathered}
\mathbb{E}\left\{\bar{\gamma}_{k+1} \mid \tilde{\xi}_{k}^{j}, \text { all } j\right\}=\frac{1}{N_{p}} \sum_{i=1}^{N_{S_{k}}}\left[\frac{N_{p}}{N_{S_{k}}} \gamma_{k+1}\left(\tilde{\xi}_{k}^{i}\right)\right] \\
\operatorname{Var}\left\{\bar{\gamma}_{k+1} \mid C_{\tilde{\xi}}^{k}\right\} \\
=\frac{1}{N_{p}^{2}} \sum_{i=1}^{N_{S_{k}}}\left[\frac{N_{p}}{N_{S_{k}}} \gamma_{k+1}\left(\tilde{\xi}_{k}^{i}\right)\left(1-\gamma_{k+1}\left(\tilde{\xi}_{k}^{i}\right)\right)\right] \\
+\frac{1}{N_{p}^{2}} \sum_{i=1}^{N_{S_{k}}}\left[\frac{\left(N_{p} \bmod N_{S_{k}}\right)}{N_{S_{k}}}\left(1-\frac{1}{N_{S_{k}}}\right)\left(\gamma_{k+1}\left(\tilde{\xi}_{k}^{i}\right)\right)^{2}\right] \\
+\frac{1}{N_{p}^{2}} \sum_{i=1}^{N_{S_{k}}} \sum_{\substack{N_{S_{k}} \\
i^{\prime}=1}}^{i^{\prime} \neq i}\left[\frac{-\left(N_{p} \bmod N_{S_{k}}\right)}{N_{S_{k}}^{2}}\left(\gamma_{k+1}\left(\tilde{\xi}_{k}^{i}\right) \gamma_{k+1}\left(\tilde{\xi}_{k}^{i^{\prime}}\right)\right)\right]
\end{gathered}
$$

Elaboration of (71) and (72) yields the equations of Proposition 4. Q.E.D.

\section{Proof of Proposition 5}

To evaluate $K_{k}^{i}, i=1,2, \ldots, N_{S_{k}}$, we define scalar parameter $\alpha_{k}$ as follows:

$$
\alpha_{k} \triangleq\left\lfloor\frac{N_{p}}{N_{S_{k}}}\right\rfloor
$$

with floor function $\lfloor x\rfloor \triangleq \max \{i \in \mathbb{Z} \mid i \leq x\}$.

For Fixed Assignment Splitting, the vector $\left(K_{k}^{1}-\alpha_{k}, K_{k}^{2}-\alpha_{k}, \ldots, K_{k}^{N_{S_{k}}}-\alpha_{k}\right)$ follows a multivariate hypergeometric distribution with the number of trials equal to $\left(N_{p} \bmod N_{S_{k}}\right)$, and with equal success probabilities $\frac{1}{N_{S_{k}}}$. Using multivariate hypergeometric distribution properties, we know for $i=1,2, \ldots, N_{S_{k}}$ :

$$
\mathbb{E}\left\{K_{k}^{i}-\alpha_{k} \mid \tilde{\xi}_{k}^{j}, \text { all } j\right\}=\frac{N_{p} \bmod N_{S_{k}}}{N_{S_{k}}}
$$




$$
\begin{gathered}
\operatorname{Var}\left\{K_{k}^{i}-\alpha_{k} \mid \tilde{\xi}_{k}^{j}, \text { all } j\right\} \\
=\left(N_{p} \bmod N_{S_{k}}\right) \frac{\left[N_{S_{k}}-\left(N_{p} \bmod N_{S_{k}}\right)\right]}{N_{S_{k}}-1} \frac{1}{N_{S_{k}}}\left(1-\frac{1}{N_{S_{k}}}\right) \\
=\frac{\left(N_{p} \bmod N_{S_{k}}\right)}{N_{S_{k}}} \frac{\left[N_{S_{k}}-\left(N_{p} \bmod N_{S_{k}}\right)\right]}{N_{S_{k}}}
\end{gathered}
$$

From (74) and (75), we can derive:

$$
\mathbb{E}\left\{K_{k}^{i} \mid \tilde{\xi}_{k}^{j}, \text { all } j\right\}=\frac{N_{p} \bmod N_{S_{k}}}{N_{S_{k}}}+\alpha_{k}=\frac{N_{p}}{N_{S_{k}}}
$$

and

$$
\operatorname{Var}\left\{K_{k}^{i} \mid \tilde{\xi}_{k}^{j}, \text { all } j\right\}=\frac{\left(N_{p} \bmod N_{S_{k}}\right)}{N_{S_{k}}} \frac{\left[N_{S_{k}}-\left(N_{p} \bmod N_{S_{k}}\right)\right]}{N_{S_{k}}}
$$

For $K_{k}^{i}$ and $K_{k}^{i^{\prime}}\left(i \neq i^{\prime}\right)$, we derive:

$$
\begin{gathered}
\operatorname{Cov}\left\{\left(K_{k}^{i}\right)\left(K_{k}^{i^{\prime}}\right) \mid \tilde{\xi}_{k}^{j}, \text { all } j\right\} \\
=\operatorname{Cov}\left\{\left(K_{k}^{i}-\alpha_{k}\right)\left(K_{k}^{i^{\prime}}-\alpha_{k}\right) \mid \tilde{\xi}_{k}^{j}, \text { all } j\right\} \\
\stackrel{a}{=}-\left(N_{p} \bmod N_{S_{k}}\right) \frac{\left[N_{S_{k}}-\left(N_{p} \bmod N_{S_{k}}\right)\right]}{N_{S_{k}}-1} \frac{1}{N_{S_{k}}} \frac{1}{N_{S_{k}}}
\end{gathered}
$$

where equality (a) holds because of the multivariate hypergeometric distribution property on the covariance.

Substituting (76) into (51) and substituting (76-78) into (53) yield:

$$
\mathbb{E}\left\{\bar{\gamma}_{k+1} \mid \tilde{\xi}_{k}^{j}, \text { all } j\right\}=\frac{1}{N_{p}} \sum_{i=1}^{N_{S_{k}}}\left[\frac{N_{p}}{N_{S_{k}}} \gamma_{k+1}\left(\tilde{\xi}_{k}^{i}\right)\right]
$$




$$
\begin{gathered}
\operatorname{Var}\left\{\bar{\gamma}_{k+1} \mid C_{\tilde{\xi}}^{k}\right\} \\
=\frac{1}{N_{p}^{2}} \sum_{i=1}^{N_{S_{k}}}\left[\frac{N_{p}}{N_{S_{k}}} \gamma_{k+1}\left(\tilde{\xi}_{k}^{i}\right)\left(1-\gamma_{k+1}\left(\tilde{\xi}_{k}^{i}\right)\right)\right] \\
+\frac{1}{N_{p}^{2}} \sum_{i=1}^{N_{S_{k}}}\left[\frac{\left(N_{p} \bmod N_{S_{k}}\right)}{N_{S_{k}}} \frac{\left[N_{S_{k}}-\left(N_{p} \bmod N_{S_{k}}\right)\right]}{N_{S_{k}}}\left(\gamma_{k+1}\left(\tilde{\xi}_{k}^{i}\right)\right)^{2}\right] \\
+\frac{1}{N_{p}^{2}} \sum_{i=1}^{N_{S_{k}}} \sum_{i^{\prime}=1}^{N_{S_{k}}}\left[\frac{-\left(N_{p} \bmod N_{S_{k}}\right)}{N_{S_{k}}^{2}} \frac{\left[N_{S_{k}}-\left(N_{p} \bmod N_{S_{k}}\right)\right]}{N_{S_{k}}-1}\right. \\
\left.\cdot\left(\gamma_{k+1}\left(\tilde{\xi}_{k}^{i}\right) \gamma_{k+1}\left(\tilde{\xi}_{k}^{i^{\prime}}\right)\right)\right]
\end{gathered}
$$

Elaboration of (79) and (80) yields the equations of Proposition 5. Q.E.D.

\section{References}

Abate A, D'Innocenzo A, Di Benedetto MD (2011) Approximate abstractions of stochastic hybrid systems. IEEE Trans Autom Control 56(11):2688-2694

Alur R, Henzinger T, Laffierriere G, Pappas G (2000) Discrete abstractions of hybrid systems. Proc IEEE 88:971-984

Asmussen SH, Albrecher H (2010) Ruin probabilities. In: Advanced series on statistical science \& applied probability, 2 nd edn, vol 14. World Scientific Publishing Co. Pte. Ltd., Hackensack

Bowsher CG, Swain PS (2012) Identifying sources of variation and the flow of information in biochemical networks. Proc Natl Acad Sci USA 109(20):E1320-E1328

Blom HAP, Krystul J, Bakker GJ (2006) A particle system for safety verification of free flight in air traffic. In: Proceedings of IEEE Conference on Decision and Control, San Diego, CA, pp 1574-1579

Blom HAP, Krystul J, Bakker GJ (2007) Free flight collision risk estimation by sequential MC simulation. In: Cassandras CG, Lygeros J (eds) Stochastic hybrid systems. Taylor \& Francis, New York, pp 249-281

Bollobás B (2001) Random graphs, volume 73 of Cambridge Studies in Advanced Mathematics. Cambridge University Press, Cambridge, second edition

Botev ZI, Kroese DP (2008) An efficient algorithm for rare-event probability estimation, combinatorial optimization, and counting. Methodol Comput Appl Probab 10(4):471-505

Bucklew JA (2004) Introduction to rare event simulation. Springer, New York

Cérou F, Legland F, Del Moral P, Lezaud P (2005) Limit theorems for the multilevel splitting algorithm in the simulation of rare events. In: Proceedings of Winter Simulation Conference. Orlando, FL, pp 682-691

Cérou F, Del Moral P, Legland F, Lezaud P (2006) Genetic genealogical models in rare event analysis. Latin Am J Probab Math Stat 1:181-203

Del Moral P (2004) Feynman-Kac formulae: genealogical and interacting particle systems with applications. Springer, New York

Del Moral P, Kouritzin M, Miclo L (2001) On a class of discrete generation interacting particle systems. Electron J Probab 6:1-26

Di Benedetto MD Di Gennaro S, D’Innocenzo A (2015) Hybrid Systems and Verification by Abstraction, in Hybrid Dynamical Systems, Springer, Switzerland, M. Djemai and M. Defoort (eds.), Lecture Notes in Control and Information Sciences 457, pp. 1-25

Garvels MJ, Kroese DP (1998) A comparison of RESTART implementations. In: Proceedings of the 30th conference on Winter simulation, pp 601-608. IEEE Computer Society Press

Garvels MJJ (2000) The splitting method in rare event simulation. PhD Thesis, University of Twente, The Netherlands 
Gerber M, Chopin N, Whiteley N (2019) Negative association, ordering and convergence of resampling methods. Ann Stat 47(4):2236-2260

Glasserman P, Heidelberger P, Shahabuddin P, Zajic T (1998) A large deviations perspective on the efficiency of multilevel splitting. IEEE Trans Autom Control 43(12):1666-1679

Glasserman P, Heidelberger P, Shahabuddin P, Zajic T (1999) Multilevel splitting for estimating rare event probabilities. Oper Res 47:585-600

Julius AA, Pappas GJ (2009) Approximations of stochastic hybrid systems. IEEE Trans Autom Control 54(6):1193-1203

Karlin S, Taylor HM (1975) Brownian motion. In: Karlin S, Taylor HM (eds) A first course in stochastic processes. Academic Press, New York, pp 340-391

Krystul J (2006) Modelling of stochastic hybrid systems with applications to accident risk assessment, PhD Thesis, University Twente, Enschede

L'Ecuyer P, Demers V, Tuffin B (2006) Splitting for rare-event simulation. In: Proceedings of the 38th conference on Winter simulation. Winter Simulation Conference. Monterey, California, pp. 137-148

L'Ecuyer P, Demers V, Tuffin B (2007) Rare events, splitting, and quasi-Monte Carlo. ACM Trans Model Comput Simul TOMACS 17(2):9

L'Ecuyer P, LeGland F, Lezaud P, Tuffin B (2009) Splitting techniques. In: Rubino G, Tuffin B (eds) Rare event simulation using Monte Carlo methods. Wiley, New York, pp 39-61

Morio J, Balesdent M (2016) Estimation of rare event probabilities in complex aerospace and other systems. Woodhead Publishing, Swaston

Prandini M, Hu J (2007) Stochastic reachability: theory and numerical approximation. In: Cassandras CG, Lygeros J (eds) Stochastic hybrid systems. Taylor \& Francis, New York, pp 107-137

Robert P (2003) Stochastic networks and queues, volume 52 of Applications of Mathematics. Springer, New York, french edition. Stochastic Modelling and Applied Probability

Rubinstein R (2010) Randomized algorithms with splitting: why the classic randomization algorithms do not work and how to make them work. Methodol Comput Appl Probab 12:1-50

Tuckwell HC, Wan FY (1984) First-passage time of Markov processes to moving barriers. J Appl Probab 21(4):695-709

Publisher's Note Springer Nature remains neutral with regard to jurisdictional claims in published maps and institutional affiliations. 